\title{
Le serpent léontocéphale Chnoubis et la magie de l'époque romaine impériale
}

\author{
Véronique DASEN \\ Université de Fribourg \\ Institut des sciences de l'Antiquité et du monde byzantin \\ 16, rue Pierre-Aeby CH- 1700 Fribourg (Suisse) \\ v.dasen@bluewin.ch \\ Árpád M. NAGY \\ Musée des Beaux-Arts, Budapest \\ Collection des Antiquités \\ 41, rue Gy. Dózsa H- 1396 Budapest (Hongrie) \\ amnagy@szepmuveszeti.hu
}

MOTS CLÉS

gemmes

magie

décans

Chnoubis

astrologie

mélothésie

médecine

KEY WORDS

Gems

Magic

Decans

Chnoubis

Astrology

Melothesia

Medicine
Dasen V. \& Nagy Á. M. 2012. Le serpent léontocéphale Chnoubis et la magie de l'époque romaine impériale. Anthropozoologica 47.1 : XX-XX.

Le serpent à tête de lion radiée, désigné par le nom Chnoubis ou Chnoumis, est l'une des figures familières des gemmes magiques de l'époque romaine impériale. Différentes traditions - médicales, astrologiques, lapidaires - attribuent à cette créature diverses compétences thérapeutiques (contre les maux d'estomac, pour la protection de la matrice...). Cet article dresse un bilan de l'iconographie de Chnoubis/Chnoumis qui apparaît sur différentes sortes de pierre, souvent de forme arrondie, et examine la signification et la fonction du "signe de Chnoubis " une haste barrée de trois $S$, qui lui est associé. Nous ferons aussi le point des différentes hypothèses concernant l'origine du personnage, notamment sur ses rapports avec Knem, le premier décan du lion, le dieu primordial Kematef (Kmeph), le dieu bélier Khnoum et les divinités solaires, ainsi qu'avec d'autres serpents comme l'Agathodaimon. Cette enquête nous permettra d'expliquer comment une entité décanique est devenue une puissante divinité solaire à part entière, associée au Dieu d'Israël, dont la popularité survit à l'Antiquité.

\section{ABSTRACT}

The lion-headed snake Chnoubis and Roman imperial magic

The radiated and lion-headed snake, designated by the name Chnoubis or Chnoumis, is one of the familiar figures of magical gems dating to the Roman imperial period. Different traditions - medical, astrological, relating to the quality of stones - attribute to this creature various therapeutic competences (against stomach troubles, for the protection of the uterus). This paper reviews the iconography of Chnoubis/Chnoumis which appears on different types of stones, often of a round shape, and examines the meaning and function of the so-called "Chnoubis sign", a triple S with a horizontal stroke in the middle, often associated wirth the snake. We also review the different hypotheses relating to the origin of the figure, especially its relationship with Knem, the first decan of the lion, the primordial god Kematef (Kmeph), the ram-god Khnum and solar deities, as well as with other snakes like the Agathodaimon. This survey allows us to suggest how a decanic entity became a powerful solar deity, associated with the God of Israel, whose popularity survived Antiquity. 
Dasen V. \& Nagy Á. M.

\section{INTRODUCTION}

\section{L'ÉTAT DES RECHERCHES}

Peu connu des antiquistes, le dieu Chnoubis occupe un rôle éminent sur les gemmes dites magiques de l'époque romaine impériale qui constituent une catégorie bien définie d'intailles dans la glyptique romaine ${ }^{1}$. Ces gemmes se distinguent des types " ordinaires " à trois niveaux. D'une part, elles comportent des voces magicae ou logoi, des formules magiques incompréhensibles d'origine diverse, gravées en lettres grecques, auxquelles s'ajoutent des charactères ou signes magiques. Enfin, leurs images suivent des schémas iconographiques particuliers, puisant souvent dans différents répertoires, principalement gréco-romain et égyptien, ou avec de nouveaux types spéciaux, comme la divinité anguipède à tête de coq ou le serpent léontocéphale Chnoubis.

Deux caractéristiques structurelles s'ajoutent aux caractéristiques formelles. Les pierres sont souvent gravées aussi sur le revers, parfois même sur le biseau. Les textes ne sont pas écrits de manière rétrograde, de façon à servir de sceau, comme sur la plupart des intailles "normales", mais l'inscription est destinée à être lue directement sur la gemme : c'est l'intaille même, en tant qu'amulette, qui fonctionne comme une sphragis, un sceau, et témoigne des rapports spéciaux du mage avec la sphère divine.

Les Zaubergemmen ne sont toutefois qu'un type de talismans parmi d'autres de l'époque impériale (Nagy 2011 et sous presse). Leur datation précise est problématique en l'absence quasi totale d'éléments de chronologie livrés par le contexte archéologique ou l'étude du style. En recoupant les sources littéraires et l'histoire de la glyptique romaine, on estime que leur production a débuté au $\mathrm{I}^{\text {er }}$ siècle av. J.-C. et qu'elle a connu son apogée aux $\mathrm{II}^{\mathrm{e}}$ et $\mathrm{III}^{\mathrm{e}}$ siècles ap. J.-C.

1. Les principales études sur le sujet : Bonner 1950 : spéc. 54-60 sur Chnoubis ; Michel 2004 : 165-170 ; Zwierlein-Diehl 2007 : 224-225; Dasen, 2011b. Sur Chnoubis, voir en dernier lieu Mastrocinque 2008b ; Vitellozzi 2010 : 397-398.
Leur mode de diffusion, ainsi que l'identité de leurs utilisateurs, restent à définir. La question de l'identification des pièces post-antiques n'est également pas encore résolue.

La place qu'occupe la figure du serpent léontocéphale Chnoubis parmi les gemmes magiques est particulièrement importante. Dans le cadre du projet Ophiaka, nous avons commencé à réunir les sources écrites et iconographiques qui s'y rapportent. Son importance numérique n'a d'égal que le schéma dit de la divinité anguipède alectorocéphale. Dans le corpus d'environ trois mille pièces rassemblées par Simone Michel, plus de trois cents gemmes se rapportent à Chnoubis (Michel 2004 : 255263, \$11 Chnoubis). Dans sa monographie sur les décans égyptiens, Joachim F. Quack livre un nouveau catalogue exhaustif de près de 400 gemmes dont il propose une interprétation minutieuse et très détaillée ; nous lui sommes très reconnaissants d'avoir mis à notre disposition des chapitres de son manuscrit encore inédit (Quack, sous presse : ch. 2.3.3). Depuis les travaux pionniers de H. Philipp et d'E. Zwierlein-Diehl (1986; 1992), appliquant aux gemmes magiques les méthodes d'analyse de l'archéologie classique, de nombreuses pistes de recherches ont été explorées, mais l'étude reste ardue en l'absence de bonnes publications photographiques des pièces ${ }^{2}$.

Les trois objectifs que nous avons cherchés à atteindre dans cet article sont les suivants :

I. Mieux comprendre la figure de cette divinité sur les gemmes magiques.

II. Analyser ses fonctions attestées par différentes sources.

III. Questionner, par une analyse parallèle du texte et de l'image, les rapports entre cette divinité et d'autres figures divines afin de saisir l'étendue de son influence à l'époque impériale.

2. La banque de données des gemmes magiques en construction à Budapest permettra bientôt d'actualiser régulièrement ces données. The Campbell Bonner database (désormais abrégée CBd-) <http://classics.mfab.hu/talismans/>. 
En conclusion, nous tenterons d'établir ce qui caractérise les amulettes avec Chnoubis et de poser quelques jalons chronologiques. S’il est impossible, dans l'état de nos connaissances, de proposer une reconstruction historique du développement de cette figure divine, nous ferons quelques hypothèses sur la formation du type et sa fonction en terminant avec de nouveaux documents qui témoignent de la survie post-antique de Chnoubis.

\section{QUELQUES ÉLÉMENTS DE TYPOLOGIE}

Chnoubis est une figure aisément identifiable dans sa forme la plus répandue sur les gemmes magiques ${ }^{3}$. Il est d'ordinaire représenté comme un serpent à tête de lion, le bas du corps enroulé sur lui-même en formant une ou plusieurs boucles, la tête redressée, radiée de cinq à douze rayons (Fig. 1a, 5b) et/ou nimbée (Fig. 8, 9b). Plus rarement, son corps peut être entièrement allongé, sans circonvolutions (Fig. 6), parfois stylisé à l'extrême. Sur l'intaille en verre d'Avenches, Chnoubis est ainsi réduit à l'essentiel : un corps allongé serpentiforme avec une tête filiforme couronnée de huit rayons (Fig. 2) ${ }^{4}$. Des variantes le dotent d'une tête ou d'un corps humains (Fig. 3a ; SGG 2008 : Aq 9). La frontière entre les figures très similaires de l'Agathodaimon et d'autres serpents à tête radiée est actuellement impossible à tracer ${ }^{5}$, tandis que deux variantes restent aussi à interpréter : la figure léontocéphale à tête radiée qui tient parfois un Chnoubis dans la main et le groupe des gemmes sur lesquelles Chnoubis est face à un quadrupède ${ }^{6}$.

3. À côté du catalogue iconographique de Quack, sous presse: ch. 2.3.3, voir les classifications du LIMC (Kiss 1986), von Lieven 2000 et surtout Michel 2004 : 255-263, \$11. Chnoubis.

4. Guisan 1975: 11, n 1.16, pl. 2, 16 (= CBd-1021 ; d'après le contexte de l'insula, datation de la première moitié du III' siècle).

5. Voir p. ex. une gemme de Vérone : SGG 2008 : 188, Vr 7 : Agathodaimon; Quack, sous presse : ch. 2.3.3 : Chnoubis. Voir aussi Michel 2004 : 262-263, \$11.9.

6. Pour les différentes interprétations voir von Lieven $2000: 29$, n. 43 et Mastrocinque $2002: 109$, n. 1.

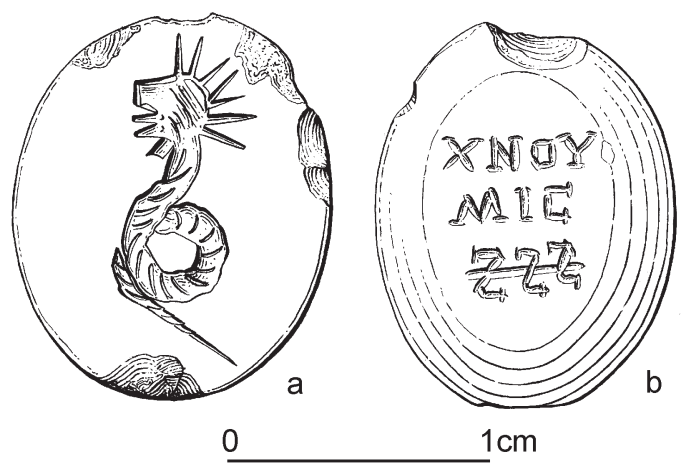

FIG. 1a \& b.- Jaspe vert. Londres, British Museum inv. G 397, EA 56397. D’après Michel 2001a : nº 305.

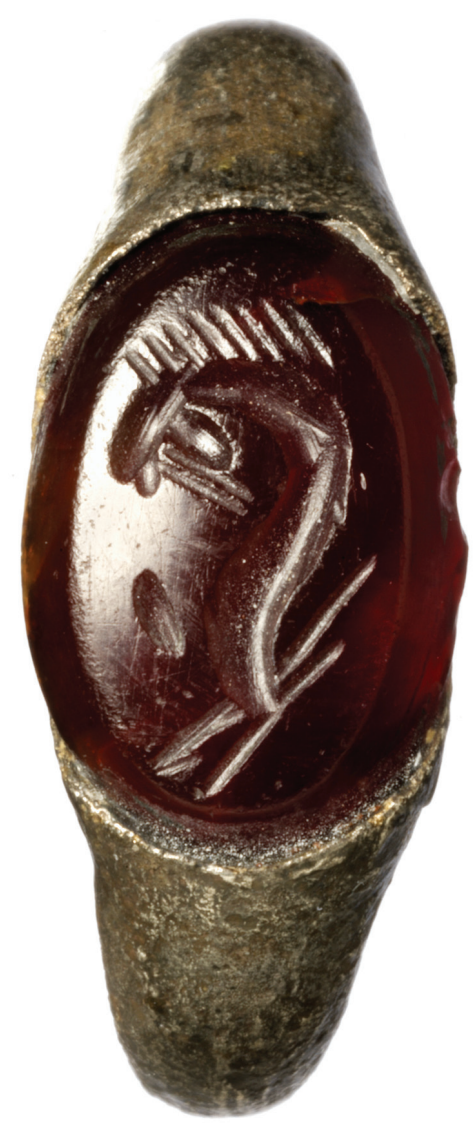

FIG. 2.- Verre rouge serti dans une bague en argent. D’Avenches, insula 16. Avenches, Musée romain, inv. 66/9831. Photo Musée romain. 
Il faut souligner que le motif du serpent léontocéphale à tête radiée et/ou nimbée apparaît quasi-exclusivement sur les gemmes magiques - à quelques exceptions près que nous présenterons plus loin ${ }^{7}$. Cette figure est accompagnée du nom Chnoubis (parfois Chnoumis, Chnouphis) (Fig. 1b, 8, 9b) dont nous examinerons la signification. Le nom est composé de sept lettres qui semblent avoir une valeur numérologique ; le total donne 1332, c'està-dire $3 \times 444$, un triple 4, chiffre sacré qui correspond au Tetragrammaton $\mathrm{YHWH}^{8}$. On relèvera l'absence de ce nom dans les papyrus magiques et les amulettes en métaux précieux où il n'apparaît que comme élément de mots composés (Kotansky 1994). Son absence des papyrus magiques est d'autant plus remarquable que ces papyrus offrent de nombreuses correspondances avec les gemmes ${ }^{9}$. La figure du serpent léontocéphale est également associée à un signe magique, une haste barrée de trois $S$, conventionnellement appelé "signe de Chnoubis" (Fig. 1b, 3b, 8, 9b). Ce signe occupe une place à part parmi les charactères magiques, car son origine est connue, contrairement aux autres charactères : il provient des représentations égyptiennes du décan Kenmet. Dans sa forme originelle, il s'agit d'un serpent dressé qui porte horizontalement trois autres serpents de taille inférieure ${ }^{10}$.

Le type du support est relativement constant. Le serpent est d'ordinaire gravé sur des pierres trans-

7. Comme J. F. Quack (sous presse), nous ne pensons pas que les monnaies du III $^{\mathrm{e}}$ siècle frappées en Moésie (Nicomédie) représentent Chnoubis (pace Drexler 1924-1937 : 859-861). Les serpents sont parfois nimbés ou peuvent avoir des rayons autour de la tête, mais ils n'ont jamais de tête de lion. Sur le type iconographique, voir p. ex. Vismara $2000: 108, n^{\circ} 432$. Nous remercions Wolfgang Fischer-Bossert (Berlin) d'avoir bien voulu nous aider à interpréter ces monnaies.

8. Zwierlein-Diehl 2007 : 224. Abrasax constitue un autre exemple bien connu d'isopséphie.

9. Sur ces rapports, voir Nagy 2002a : spéc. 157 et 177-179 (avec la liste des gemmes proposées par les papyrus magiques).

10. Von Lieven $2000: 22-24$. Les gemmes où l'image n'est pas accompagnée de nom et de signe sont très rares : Michel 2001a : $\mathrm{n}^{\circ} 326$ (= CBd-712).

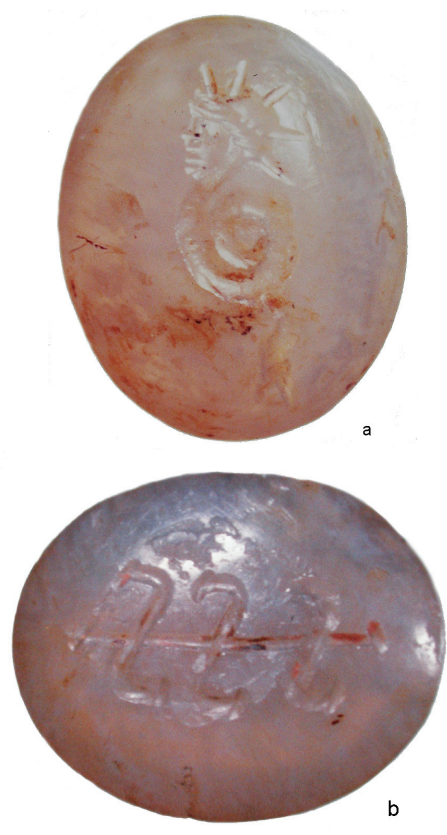

FIg. 3a \& b.- Calcédoine laiteuse. Aquileia, Museo Archeologico Nazionale inv. 26073. Photo A. Mastrocinque.

lucides de couleur verte (Fig. 1), parfois brun foncé ou noire (Fig. 9), auxquelles les textes font référence (Appendice $4-7)^{11}$, mais des exemples se trouvent aussi sur des pierres laiteuses (Fig. 3) ou de couleur rouge (Fig. 2, 5). Le signe isolé figure principalement sur des calcédoines (Quack, sous presse : ch. 2.3.3).

\section{L'IDENTITÉ DU CHNOUBIS DES GEMMES MAGIQUES}

\section{LES ANTÉCÉDENTS ÉGYPTIENS : LE DIEU KHNOUM? LE DÉCAN KENMET?}

L'analyse du nom Chnoubis ne livre pas aisément l'identité du dieu, car Chnoubis est la transcription grecque de deux divinités égyp-

11. Relevons que le iaspis des textes ne correspond pas à notre jaspe, il désigne plutôt un groupe de calcédoines. Voir p. ex. Plantzos 1999 : 36 et n. 6 ; Zwierlein-Diehl 2007 : 307-309. Sur le matériau des gemmes de Chnoubis, voir Michel 2001a : 193 ; Quack, sous presse : ch. 2.3.3. 
tiennes différentes ${ }^{12}$. La première, Khnoum, est le dieu créateur à tête de bélier, représenté sporadiquement sur les gemmes magiques. Dans un article fondateur, W. Drexler avait rejeté les rapports entre Khnoum et le Chnoubis des gemmes magiques, mais l'hypothèse est cependant toujours présente dans les milieux scientifiques ${ }^{13}$. La deuxième divinité, Kenmet, semble avoir un rôle plus important ${ }^{14}$. Elle appartient au groupe des décans égyptiens qui remontent à l'Ancien Empire. À l'époque hellénistique, les décans (en grec angeloi, daimones, prosôpa) sont clairement liés au zodiaque, composé de douze signes qui comprennent chacun trois décans de dix degrés. Ils peuvent être également considérés comme des puissances subordonnées à une divinité dont le décan - selon l'expression courante des traités astrologiques - " porte le masque " (prosôpon pherei) ${ }^{15}$. Nous proposons d'ajouter une importante gemme à ce dossier. Une amulette en calcédoine de Budapest porte gravée une série de vœux à un certain Asclépiacos afin qu'il puisse parcourir sa vie en compagnie des triades angéliques : synodeian meta tôn prôtangelôn kai mesangelôn kai teleangelôn. La métaphore du parcours commun rend évident que les noms génériques prôtangeloi, mesangeloi, teleangeloi font référence aux décans ${ }^{16}$.

On ne saurait exclure que la double étymologie du nom Chnoubis, Khnoum et Kenmet,

12. Signalons une autre spéculation étymologique qui le rapprocherait du dieu Knèph (en égyptien Km3t.f, Kematef), un serpent démiurgique lié à la cosmologie thébaine ; Delattel Derchain 1964 : 56-57. Cette interprétation a été réfutée par Thissen 1996.

13. Drexler 1890-1897 : 1260. Pour un bilan exhaustif des différentes interprétations relatives à Chnoubis, Quack, sous presse : ch. 2.3.3. Sur les rapports Khnoum - Chnoubis voir en dernier lieu, Sfameni 2010 : 451-452 ; Vitellozzi 2010 : 397-398.

14. Thissen $1992: 155$, n. 16 renvoie à Sambin $1995: 422-425$.

15. Le manuel de base sur les décans est Gundel 1936a : spéc. 30-36 sur les prosôpa ; la nouvelle synthèse est livrée par Quack, sous presse.

16. CBd-5. Voir Nagy 1992 : spéc. 104-105 sur l'interprétation des angeloi. David Jordan per litteras a corrigé la lecture : synodeian et non synouseian. Nous tenons à le remercier de la discussion du texte de la gemme. Mesangeloi et teleangeloi sont des hapax legomena. ait été exploitée par la magie de l'époque impériale dont les tendances synthétisantes sont bien connues, mais les éléments qui pourraient le confirmer manquent encore. Néanmoins, nous présenterons plus loin un argument iconographique qui nous semble témoigner des rapports entre les deux divinités sur les gemmes magiques.

\section{L'iconographie de Chnoubis dans les SOURCES LITTÉRAIRES}

À côté des textes grecs qui se rapportent au dieu Khnoum $^{17}$, six textes littéraires grecs et latins décrivent l'iconographie de Chnoubis (Appendice 1-2, 4-7). Trois œuvres le détaillent de manière très précise et livrent tous ses éléments constitutifs : le corps de serpent et la tête de lion couronnée de rayons de soleil. Il s'agit de deux écrits hermétiques, la Hiera Biblos Hermou et le Liber Hermetis Trismegistri (Appendice 1-2), où Chnoubis est clairement identifié au premier décan du signe du Lion qui régit le cœur, kardia, un terme qui peut aussi désigner l'estomac ou le ventre de manière large, et du traité sur les propriétés des pierres de Socrate et Denys (35) qui mentionne son effet thérapeutique sur l'estomac, mais sans se référer aux décans (Appendice 4) ; l'auteur spécifie qu'il est également possible de graver une protomè radiée du lion, que l'on trouve sporadiquement sur les gemmes ${ }^{18}$. Socrate et Denys (36) mentionnent une variante de Chnoubis où

17. Nos recherches ne concernent donc pas les papyrus ni les ostraca d'Éléphantine, ni les inscriptions gravées sur marbre de l'époque hellénistique. Sur les papyrus d'Éléphantine se rapportant au conflit de la communauté juive avec les prêtres de Khnoum au Ves. av. J.-C., voir Porten 1996 : 15-18, spéc. 17-18, 77-79 et 139-149. Pour les ostraca, voir Dupont-Sommer 1945 et 1947. Pour un choix d'inscriptions, Kiss 1986: 272.

18. Nous ne connaissons que deux exemplaires de cette variante : Zazoff $1970: 238, \mathrm{n}^{\circ} 165$ (= Michel 2001b : 70-71, $\mathrm{n}^{\circ}$ 70 ; SGG $2004: 252, \mathrm{n}^{\circ} 165$; Quack, sous presse : ch. 2.3.3); Zazoff $1970: 239, n^{\circ} 168$ (= SGG $2004: 253, n^{\circ} 166$; Quack, sous presse : 560 et n. 3524 . S'agit-il vraiment d'une protomè ou plutôt d'une crinière de lion ? Les deux gemmes proviennent de la collection d'Antonio Capello (1652-1729 ; voir SGG 2004 : 131-132) et pourraient être post-antiques. 
l'image d'un serpent à trois têtes doit favoriser l'allaitement (Appendice 4).

On relèvera que les traités de médecine (Appendice 5,6) décrivent le corps du serpent et les rayons, mais pas la tête de lion ; s'ils ne mentionnent pas les décans, ils reconnaissent au serpent radié, associé au iaspis, un pouvoir sur la région du ventre, même si Galien et son compilateur Aétius (Appendice 5,7) n'accordent pas d'efficacité thérapeutique au motif gravé sur la gemme, mais uniquement à la pierre et à sa couleur verte ${ }^{19}$.

Cet ensemble de sources confirment que le Chnoubis des gemmes magiques est bien un décan, comme le montrent son image, son nom et son signe. Cependant, contrairement au zodiaque, les décans ne forment pas un système clos.

\section{LES DÉCANS SUR LES GEMMES MAGIQUES}

Deux tablettes astrologiques gravées sur des diptyques en ivoire montrent l'image du serpent léontocéphale parmi les représentations des décans. Ces objets constituent un témoignage précieux sur l'iconographie zodiacale à l'époque impériale (Fig. $4 \mathrm{a}, \mathrm{b})^{20}$. Elles proviennent du sanctuaire d'Apollon Grannus à Grand (Vosges), où l'on semble avoir pratiqué l'incubation. Elles furent jetées volontairement brisées dans un puits vers 170 ap. J.-C. Probablement fabriquées en Égypte, elles devaient faire partie de l'équipement d'un astrologue qui dressait des horoscopes. On y voit gravée l'image du ciel en cercles concentriques. Au centre trônent les bustes du Soleil et de la Lune, entourés de l'image des douze signes zodiacaux,

19. Signalons un problème textuel également évoqué par Quack, sous presse : ch. 2.2.9. Dans l'édition d'Olivieri, Aétius ne décrit pas l'image à graver sur la pierre, même s'il y fait référence (Olivieri 1935 : 162 - non vidimus. Voir Appendice 7). Cependant, Drexler 1890-1897: 1262, cite le texte en latin comme suit : quidam annulis iaspidem viridem includunt et draconem radios habentem in ipsa sculpunt ex praecepto Necepsi regis, qui prosit ventriculo, "certains portent un jaspe vert en bague avec gravée l'image d'un serpent radié tel que le prescrit le roi Nechepsô ; elle est utile à l'estomac ".

20. Abry 1993 ; Bay 2008 : 100-101 ; Quack, sous presse : ch. 2.3.2.

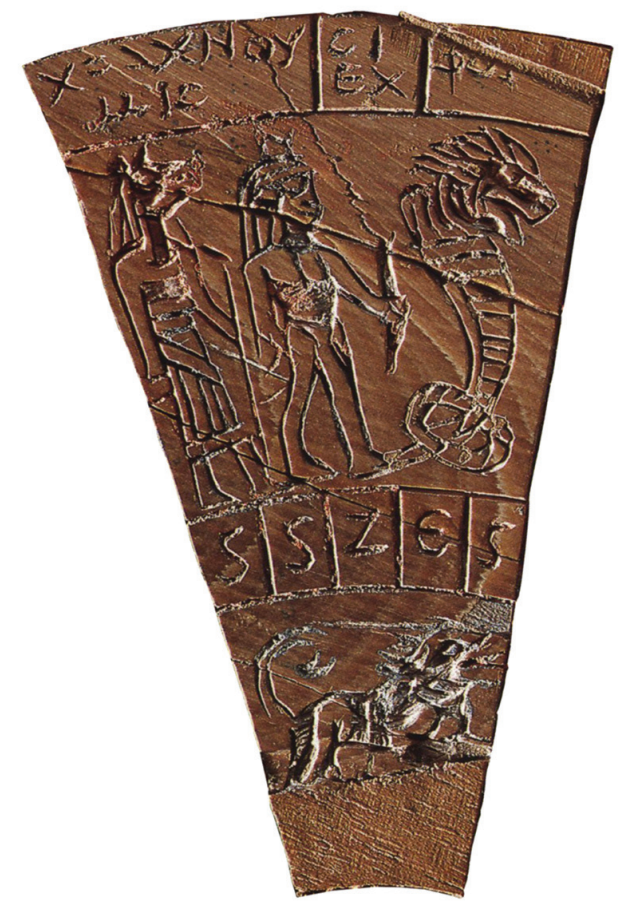

Fig. 4a.- Tablette en ivoire, de Grand, détail. Musée départemental des Vosges, Épinal, sans inv. D’après Abry 1993 : 95.

surmontés de trois figures par signe, les décans avec leurs noms. Le serpent léontocéphale apparaît dans le premier décan du Lion (Fig. 4a, b), comme le décrivent les textes hermétiques (Appendice 1-2).

Plusieurs sources indiquent que l'image des décans figurait sur des amulettes. Michel Psellos (1018-1078) fait référence à la pratique de graver sur des bagues ou anneaux " des décans aux formes variées ", dekanoi poikilomorphoi, afin d'obtenir de puissants phylaktèria ${ }^{21}$. Quelques exemples ont été identifiés ${ }^{22}$.

Nous proposons d'ajouter à la liste une gemme en cornaline conservée au musée de

21. M. Psellos, Peri paradoxôn akousmatôn, voir Duffy $1992: 112$ (cité par Gundel 1936a : 373).

22. Voir p. ex. von Lieven 2000 : 31-32 ; Michel 2001a : 107 (= CBd-507) ; 257 (= CBd-655). 
Le serpent léontocéphale Chnoubis et la magie de l'époque romaine impériale

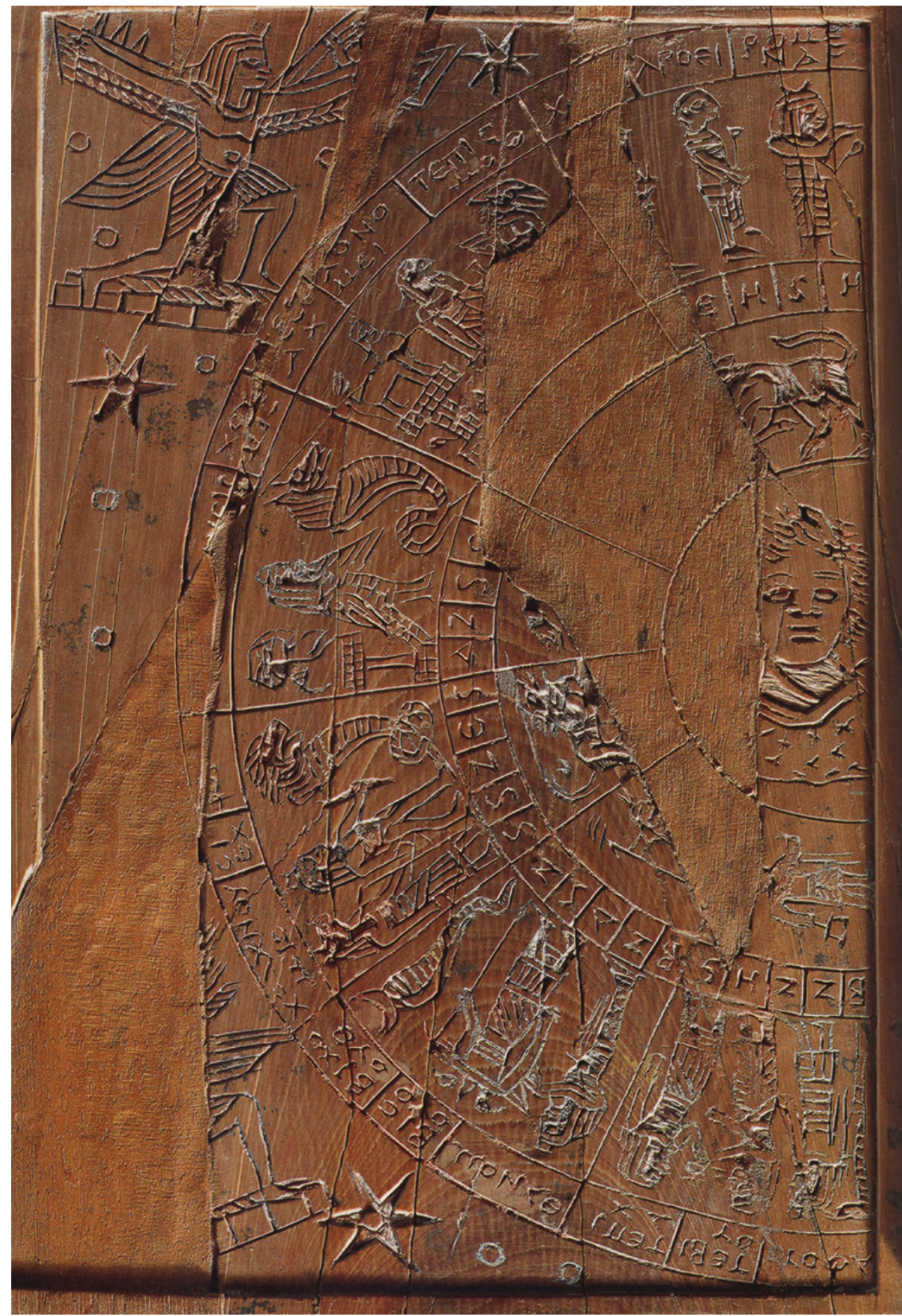

Fig. 4b.- Tablette en ivoire, de Grand. Musée départemental des Vosges, Épinal, sans inv. D’après Abry 1993 : pl. 2-3. 
Dasen V. \& Nagy Á. M.

Florence qui porte sur l'avers le type habituel de Chnoubis, mais sans nom ni signe (Fig. 5a, b ; $S G G 2008$ : 59, Fi 66). Le revers porte gravé un schéma étrange : une forme ovoïde, posée sur une petite base, au-dessus d'elle les bustes de profil de la Lune et du Soleil ; l'une avec croissant et torche, l'autre avec couronne de rayons et fouet ${ }^{23}$.

Grâce aux tablettes de Grand, nous pouvons avancer une nouvelle interprétation de cette gemme. Sur les diptyques, le nom et l'image de Chnoubis sont séparés l'un de l'autre : dans le premier décan du Lion, l'image du serpent à tête de lion est appelée Chrachnoumis. Le nom Chnoubis est inscrit dans le troisième décan du Cancer, associé à un schéma apparenté à celui de la gemme florentine : un buste double sur un socle avec piédouche ovale (Fig. 4b ; Abry 1993 : 93). Un motif semblable est décrit pour le troisième décan du Cancer, appelé Chnouphos dans la Hiera Biblos Hermou : "Celui qui a pour nom Chnouphos et pour forme deux visages de femme détournés l'un de l'autre, dont l'un (porte) un petit chapeau et l'autre un diadème. Il a le cou entouré de dragons. Toute la poitrine (le torse?) est posée sur un socle. Il régit la rate. " (trad. Ruelle 1908 : 259). Chacune des faces de la gemme florentine pourrait donc illustrer une forme d'un dieu décanique, d'un côté le Chnoubis du troisième décan du Cancer, de l'autre le Chrachnoumis léontocéphale, en train d'émerger comme entité divine à part entière ${ }^{24}$. Ce motif est pour le moment sans parallèle ${ }^{25}$, mais on peut se demander si les représentations de Chnoubis posé sur un socle de forme diverse (ciste, colonne...) ne

23. Selon A. Mastrocinque in $S G G 2008$ : 59, la forme ovoïde symboliserait l'œuf donnant vie à la divinité primordiale, alors que les deux bustes exprimeraient la nature androgyne du dieu.

24. La gemme de Florence renforce l'hypothèse de Quack, sous presse : ch. 2.3.2 : l'image du serpent léontocéphale appartenait d'abord à Chrachnoumis.

25. A. Mastrocinque in $S G G 2008: 59, \mathrm{n}$. 1, fait référence à une autre gemme, connue d'après un dessin de Gori 1736 : II, pl. 89, 5 (= Reinach $1895:$ pl. 69) seraient pas dérivées de cette iconographie décanique ${ }^{26}$.

\section{Le Chnoubis des gemmes Magiques : DÉCAN ET DIVINITÉ SOLAIRE}

La présence des décans reste cependant très rare sur les gemmes, tandis que Chnoubis est attesté sur près de 400 intailles selon le décompte réalisé par J. F. Quack. Comment expliquer cette différence ? Plusieurs chercheurs ont déjà relevé la tonalité solaire du Chnoubis des gemmes magiques $^{27}$. Nous pouvons apporter un argument iconographique qui le confirme et explique sa faveur remarquable. Sur les tablettes de Grand, comme sur les représentations pharaoniques de Kenmet, sur les autres décans de Grand comme sur les autres gemmes magiques à représentations décaniques, le serpent n'a pas de tête radiée $^{28}$. La couronne de rayons semble donc être son signe distinctif sur les gemmes magiques ; elle désigne le serpent comme une divinité solaire, bien plus qu'un simple décan ${ }^{29}$. Ce nouvel élément, spécifique aux gemmes, est une création originale qui offre l'image d'un theos hypsistos inscrite dans la théologie de l'époque impériale.

Nous avons vu que les traités de mélothésie astrologique $^{30}$, des lapidaires grecs ainsi que les textes médicaux associent la figure de Chnoubis à la protection de la région du ventre. Un petit nombre d'inscriptions confirment ce champ de compétence. Huit gemmes portent un texte qui indique leur utilisation, dont sept se réferent à

26. Michel 2004, \$ 11.4. Voir p. ex. Delatte/Derchain 1964 : $\mathrm{n}^{\circ} 77$ (colonne), $\mathrm{n}^{\circ} 79$ (ciste).

27. Voir les ouvrages cités dans la note 1 .

28. Sur l'image des décans gravés sur les gemmes magiques, voir supra note 22.

29. L'image de Chnoubis sans rayons de soleil est rarissime sur les gemmes : voir p. ex. Bonner 1950 : D 98, et les pendentifs en serpentine traités ci-dessous qui appartiennent à un groupe "périphérique »: Michel 2001a : $\mathrm{n}^{\text {os }} 409,410,411,413$ (=CBd780, 781, 136, 163). Voir également infra note 37.

30. Sur la mélothésie, qui associe à chaque décan une partie du corps, Gundel 1936a ; Quack, sous presse: ch. 2.2.9. 


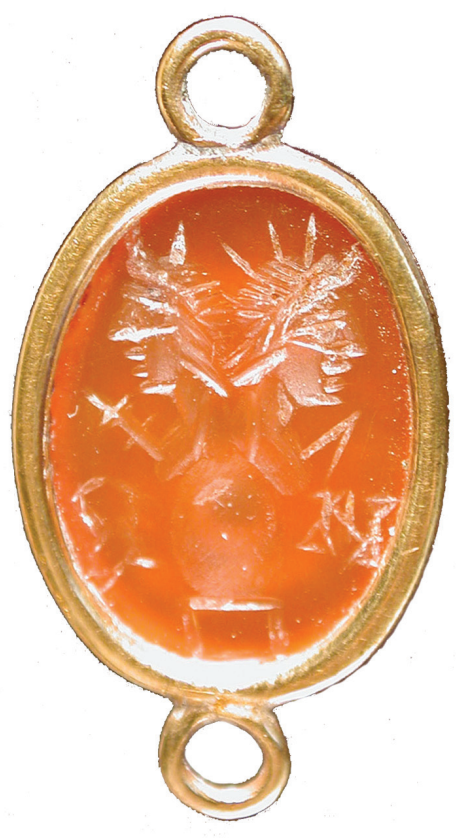

a

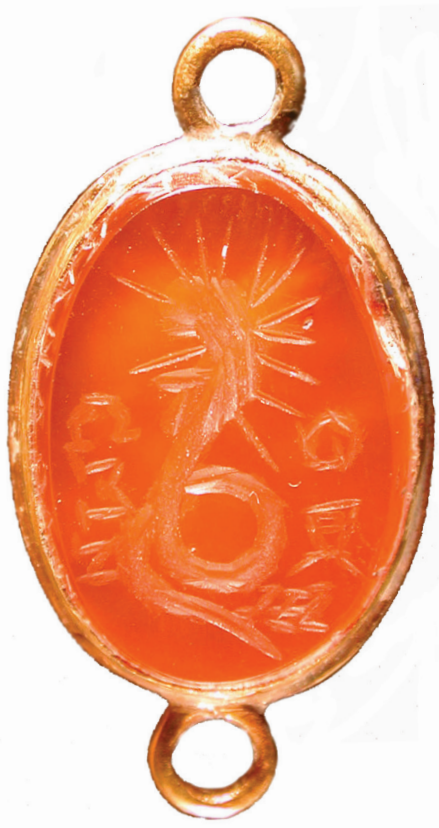

b

FIg. 5a \& b.- Cornaline. Florence, Musée archéologique inv. 15120. Photo A. Mastrocinque.

l'estomac ou à la digestion (Appendice $8 \mathrm{a}-\mathrm{g})^{31}$. À cause du nombre réduit d'exemples, des études récentes ont mis en question la fonction protectrice de Chnoubis sur l'estomac ${ }^{32}$. Mais est-il justifié de douter? La convergence remarquable entre les inscriptions des gemmes et les sources littéraires, de date et de nature différentes, ne nous apparaît pas comme une simple coïncidence. De plus, en travaillant de manière sérielle, le nombre de témoignages peut être augmenté. Une calcédoine du Cabinet des Médailles présente ainsi sur une face le serpent radié, sur l'autre le signe de Chnoubis associé à l'inscription stomachou (Appendice 8e). Une stéatite de couleur gris-blanc du Musée archéo-

31. La seule exception est une gemme publiée par Bonner 1954 : 154-157, $\mathrm{n}^{\circ} 42$. Le texte sur le revers, une prière pour faire taire des adversaires, invoque également Chnoubis. Mais cette invocation, comme Bonner l'a bien constaté, a été ultérieurement gravée sur la gemme décorée de Gorgoneion. La pièce n'appartient donc pas au corpus des gemmes Chnoubis proprement dites.

32. Michel $2004: 168$. logique de Naples porte d'un côté le serpent à la tête radiée de sept rayons, de l'autre le signe de Chnoubis associé à un triple $\mathrm{K}$ qui fait référence aux maux de ventre ${ }^{33}$.

Une pierre verte (onyx ?) résume cette équivalence : elle porte à l'avers l'image du serpent radié et au revers le signe de Chnoubis entouré d'une inscription : stomacou é Cnounis (sic), " [pour la santé] de l'estomac ou bien Chnounis »), comme si le graveur avait par erreur noté les deux termes qu'on lui avait donnés à choix ${ }^{34}$. La fonction digestive du dieu ne doit donc pas être sous-estimée, sans nier le fait que les pouvoirs de cette figure étaient plus larges, aussi dans le domaine médical, comme le suggère le champ lexical des termes qui se rapportent au ventre. Stomachos peut en effet désigner divers

33. SGG 2008 : Na 6. Sur la signification du triple K, voir Bonner 1950 : p. 63-64. Voir les variantes dans Michel 2001a, $\mathrm{n}^{\text {os }} 390-394$.

34. Mouterde 1930-31 : 74-75, fig. 8 (dessinée chez un marchand de Beyrouth ch. 2.3.3) ; Bonner $1950: 59$. 
organes avec une ouverture, comme l'utérus, la vessie, l'œsophage le larynx, une imprécision qui fait des maladies stomachiques une catégorie très large qui peut comprendre les maux utérins $^{35}$. Cette polyvalence apparaît sur une hématite de la collection Skoluda (Fig. 6), où le serpent se tient dressé à côté de l'utérus ventouse tandis que l'inscription indique qu'il apaisera les maux d'estomac (Appendice 8f) ${ }^{36}$.

Une ambiguïté similaire se trouve sur une série de pendentifs en serpentine qui portent d'un côté l'image de l'utérus-ventouse, surmonté de divinités protectrices (Anubis, Osiris, Isis, Nephtys, Chnoubis), de l'autre l'ibis qui doit apaiser ou contrôler les coliques comme l'indique l'inscription qui ordonne au revers, pesse, ou pepte "digère! ", souvent accompagné du signe de Chnoubis. Le nom d'Orôriouth, une entité présidant aux accouchements, peut être gravé, avec d'autres voces, à la place de pepte. Les deux faces de la gemme se rapportent ainsi à la géographie corporelle contrôlée par le magicien, ici les organes féminins ${ }^{37}$.

On relèvera que le Chnoubis tricéphale qui devait secourir les femmes enceintes ou allaitantes (Appendice 4) est également attesté archéologiquement. Une calcédoine de couleur laiteuse, et non noire comme prescrit par les lapidaires, porte l'image d'un serpent tricéphale, avec au revers l'inscription Chnoubi $(s)^{38}$. Comment expliquer ce contrôle du lait par le serpent ? Probablement par sa

35. Roura 1972, spéc. 320-321. Gourevitch 1976. Les trois Kappas peuvent être aussi associés aux douleurs de l'accouchement, voir Michel 2001 a : n 388 ; Dasen 2008.

36. Michel 2001b : 72-73, $\mathrm{n}^{\circ} 74$; Quack, sous presse : ch. 2.3.3 (pièce moderne).

37. Delatte/Derchain $1964: \mathrm{n}^{\text {os }} 189-190$; Michel 2001a : $\mathrm{n}^{\text {os }} 409$ 412 (= CBd-780, 781, 136, 108). Sur ce groupe de gemmes, voir Quack, sous presse : ch. 2.3.3. En outre, voir la gemme en jaspe brun conservée à Bucarest (Gramatopol 1974: 69, $\mathrm{n}^{\circ}$ 395) : "Autruche à droite, serpent à tête de lion et aigle dévorant un lapin, entourés de signes gnostiques (?) et de lettres (?). " S'agit-il d'une pièce antique? Sur cette géographie corporelle, traduite par l'image d'Heraclès et les trois K de kôlikè, voir aussi Dasen 2008.

38. Zwierlein-Diehl 1992, p. 79-80, n 18, pl. 14 ; Quack, sous presse : ch. 2.3.3.

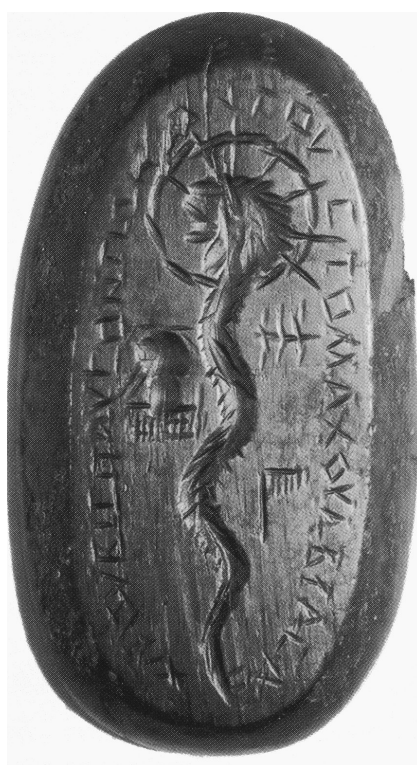

Fig. 6.- Hématite. Hambourg, Collection Skoluda M085. D’après Michel 2001b : pl. 24, n 145.

fonction de protecteur de la matrice. Le lait maternel est en effet du sang menstruel «qui a subi une coction parfaite" (Aristote, Génération des animaux, IV, 8, 777a). Le sang utérin, qui nourrissait l'embryon pendant la grossesse, se transforme en lait après la naissance ${ }^{39}$.

\section{CHNOUBIS ET LES AUTRES DIVINITÉS SUR LES GEMMES}

\section{Signe, image ET NOM}

Il est possible de préciser la nature divine et le champ de compétence de Chnoubis en analysant de plus près la présence de son signe, de son nom et de son image sur d'autres types de gemmes magiques ainsi qu'en examinant les rapports qui le lient à d'autres divinités.

39. De nombreux textes décrivent cette transformation du sang utérin en lait. Voir p. ex. Aristote, Génération des animaux, IV, 8, 776a-777a, et Plutarque, De l'amour des enfants, 495E-496A. D'origine menstruelle, le lait possède des propriétés analogues à celles du sperme ; sur ce phénomène, voir Dasen 2010. 


\section{LE SIGNE}

Sur les gemmes magiques, le signe Chnoubis (Fig. 1b, 3b, 8, 9) est presque exclusivement associé au dieu Chnoubis. Nous avons recensé dix variantes iconographiques où il est lié à d'autres figures $^{40}$ :

1. au revers d'une hématite utérine avec Seth/ Anubis ${ }^{41}$,

2. au revers d'une gemme avec le lézard et vox magica sur l'avers ${ }^{42}$,

3. sur des gemmes appartenant au groupe $A n$ guipes - Leontokephalos ${ }^{43}$,

4. au revers d'une hématite représentant Asclépios et Hygie ${ }^{44}$,

5. au revers d'hématites avec le phénix et portant l'impératif pepte, " digère $!^{45}$ ",

40. Quack, sous presse : ch. 2.3.3, livre une liste de 52 gemmes où le signe se retrouve sans le nom ni l'image de Chnoubis. À ajouter : gemme en chalcédoine. Budapest, Musée des BeauxArts, 62.21.A = CBd-152. Voir également la gemme du Cabinet des Médailles « Seyrig not in Bonner 2 " mentionnée dans la note 83.

41. Michel 2004 : pl. 72, 1 (Seth-Anubis, matrice " ailée ", voces, charactères).

42. Paris, Cabinet des Médailles, 2193 (= Delatte/Derchain 1964 : n³ 367 ; SGG 2004 : 410, n³81).

43. Anguipes : $S G G$ 2008, $\mathrm{Na} 13$; probablement aussi Michel 2001a : nº 211 (= CBd-609) avec une sorte d'anagramme du nom Chnoubis? et $n^{\circ} 396$ (= CBd-767), fragmentaire, avec au revers l'inscription pepte, "digère ! ". Leontokephalos : de Ridder 1911 : 771, $\mathrm{n}^{\circ}$ 3456, pl. 29., sur le revers : stomachou "pour l'estomac »). Madrid, Casal Garcia : 186-187, n 496 (= Quack, sous presse : 607, n. 4048), sur le revers : pepte, « digère!" 44. Delatte/Derchain $1964: \mathrm{n}^{\circ}$ 235. Cf. Fig. 7 a, b, même association avec Asclépios et Hygie, mais avec Chnoubis cette fois.

45. Nagy 1992a : 162-169, avec les pièces suivantes : Budapest, Musée des Beaux-Arts, 55.154 (= CBd-2 ; Ann Arbor, Taubman Medical Library, Bonner 29 (= Bonner 1950 : D 103. Nous remercions Mme Barbara Shipman d'avoir rendu possible l'étude de la gemme) ; ex Coll. Sarrafian, Beyrouth (= Bonner 1950 : D 104) ; La Haye, Cabinet Royal des Monnaies 1471 (= Maaskant-Kleibrik 1978 : 357, $\left.\mathrm{n}^{\circ} 1125\right)$. À ajouter : Michel 2001a, n 401 (= CBd-772) et Michel 2001b : 83-84, $\mathrm{n}^{\circ}$ 89. Nous signalons aussi la gemme Bonner 1950 : D 264, écho lointain de cette série iconographique : sur l'avers, une figure à double tête de serpent et d'ibis; elle se tient debout sur une sphère posée sur un crocodile (ces éléments se retrouvent également sur les gemmes avec le phénix) ; sur le revers, le nom et le signe de Chnoubis et l'impératif pes(s)e! (Quack, sous presse : ch. 2.3.3, doute cependant de son authenticité : « eine nicht ganz unverdächtige Gemme »).
6. sur des hématites avec l'ibis (avec Chnoubis au revers), également de fonction digestive ${ }^{46}$, 7. sur le revers d'un jaspe rouge avec Omphale, donc de vertu gynécologique ${ }^{47}$,

8. sur une hématite du type Moissonneur, donc destinée à guérir les rhumatismes ${ }^{48}$,

9. sur des hématites avec Salomon ${ }^{49}$,

10. sur des gemmes gravées de listes de charactères ${ }^{50}$.

On relèvera que les groupes 1, 4-7 concernent la santé en général ou le domaine digestif et gynécologique; des pièces du groupe 3 et les variantes 5-6 sont également liées au domaine digestif ; 2 concerne l'ophthalmie, 8 protège de l'arthrose. Dans le groupe 10, le signe Chnoubis entre dans une longue liste de charactères; sa présence ne semble pas être l'œuvre du hasard, mais rien ne permet d'en dire davantage. Excepté les groupes 2, 9 et 10, tous les types se rapportent donc explicitement à des vertus curatives qui appartiennent au domaine du ventre (kardia) régi par Chnoubis.

L'existence d'un système, où la figure et le signe de Chnoubis ont une valeur thérapeutique, est confirmée par le fait que le signe Chnoubis est absent des autres types de gemmes magiques ( $p$. ex. Aphrodite ou Horus). Il peut être utilisé seul, car son pouvoir agit également sans l'image ni le nom de Chnoubis ${ }^{51}$. Selon Marcellus Empiricus, il suffit de porter le signe autour du cou pour calmer une pleurésie ou des maux de hanches

46. Bonner 1950 : D 80 (= Michel 2004 : pl. 74, 3 ; Michel 2001a, 407 (= CBd-778). Sur ces gemmes, le signe est composé de deux $S$ barrés, variante qu'on retrouve parfois avec l'image de Chnoubis, voir p. ex. Michel 2001b : $257 \mathrm{n}^{\circ}$ 408) ; Michel 2001a : 407 ; Michel 2001b : 113, $\mathrm{n}^{\circ} 127$ (= Michel 2004 : pl. 74, 2).

47. Sur la gemme de la collection Southesk, Dasen 2008 : p. 270 , fig. 5 .

48. Michel 2001a : $\mathrm{n}^{\circ} 427$ (=CBd-785); peut-être également, avec un signe déformé Michel 2001a, nº 428 (= CBd-786).

49. Bonner 1950 : D 296 ; Michel 2001a : nº 431 (=CBd-789); Michel 2001b : $\mathrm{n}^{\circ} 107$.

50. Philipp $1986: \mathrm{n}^{\circ} 193$ (signe Chnoubis avec cinq $\mathrm{S}$ barrés); Michel 2004 : 289, $\$ 28.2 . c$, pl. 99, 1 ; $\$ 28.13 . b$, pl. 100, 3 ; SGG 2008 : Aq 28-29.

51. Voir supra, note 40 
(Appendice 6). Il apparaît aussi sporadiquement sur d'autres supports, comme les phylactères ${ }^{52}$, où il a pu détenir une valeur thérapeutique ou plus largement protectrice ${ }^{53}$, une fonction que nous pouvons supposer pour les groupes 9 et 10 .

\section{LE NOM}

Le nom de Chnoubis est absent des autres sources de la magica. On relèvera que sur les intailles magiques, le nom ne se rencontre qu'avec l'image ou le signe de Chnoubis, jamais avec d'autres divinités ${ }^{54}$. Le nom semble donc bien désigner une divinité spécifique, et pas une parole magique, dont l'utilisation peut être indépendante de la divinité, comme on le suppose pour Iaô ou Abrasax qui semblent avoir souvent la valeur de voces magicae, distincte de leur contexte original.

Il est important de souligner que le nom des divinités du panthéon gréco-égyptien n'est en principe jamais clairement inscrit sur les gemmes magiques; on n'y trouve pas «Isis », ou "Artémis ». Quel sens supplémentaire apportait à l'image l'indication du nom de Chnoubis ? La double nature de Chnoubis, à la fois décan et divinité solaire, nous amène à suggérer que, dans la plupart des cas, son nom ne constitue pas tant « le » nom d'une divinité, mais plutôt un nom secret apte à l'invoquer, car il définit l'espace astrologique dans lequel son pouvoir divin se manifeste pour faire réussir une praxis magique $^{55}$.

52. Voir p. ex. P. Berol. 21165 : Daniel - Maltomini 1990 : 2629 ; deux lamellae conservées à Oxford : Kotansky/Naveh/Shaked 1992 : 6 et Kotansky $1994: 13-15, n^{\circ} 3$ (signe avec quatre $S$ barrés) ; lamella, Rome : Morigi 2006: 165 (avec bibliographie antérieure).

53. Cf. PGM IV, 1264 : "Pour chasser un démon, aie un phylactère avec ce signe: un $S$ barré.» Relevons que le revers d'un lapis-lazuli ( $S G G 2004,92$, fig. 28) porte le signe associé à un scorpion; le signe pourrait avoir ici une valeur apotropaïque.

54. La gemme Bonner 1950 : D 264 mentionnée dans la note 45 pourrait constituer une exception, mais la documentation disponible est insuffisante pour se prononcer sur l'authenticité de la pièce (seule la photo de l'avers est publiée).

55. Le groupe des gemmes avec Aphrodite constitue peut-être un cas analogue : l'image est accompagnée du mot Arrôriphrasis,

\section{UN DIEU SANS HISTOIRE ?}

Quelques gemmes portent pourtant l'inscription anoch Chnoubi, "Je suis Chnoubis ", qui fait apparaître la divinité sous une forme personnelle, mais sans apporter de précision sur ce dieu sans mythe connu ${ }^{56}$. Sur une série de gemmes, le serpent Chnoubis est toutefois qualifié de gigantorèktès, barophitès, " qui détruit les géants ou les serpents ». Ces épiclèses semblent inscrire la figure de Chnoubis dans une narration où il serait le protagoniste d'un récit. On en déduit généralement que Chnoubis a été rapproché du Dieu d'Israël, "Destructeur des Géants ", mais aucun argument irréfutable ne le prouve $^{57}$. Les gemmes sur lesquelles Chnoubis est confronté à un quadrupède pourraient aussi être interprétées comme les représentations d'une narration inconnue.

\section{L'IMAGE}

La figure du serpent léontocéphale à tête radiée est toujours désignée sur les gemmes par le nom de Chnoubis, à quelques exceptions près que nous verrons plus bas. Son absence quasi totale des autres supports de la magica suggère qu'il est associé à des praxeis qui utilisent spécifiquement les intailles à l'intérieur du monde plus vaste de la magie gréco-égyptienne.

\section{LE RÉSEAU ICONOGRAPHIQUE}

Dans quel réseau iconographique s'inscrit la figure du serpent léontocéphale radié ou nimbé des gemmes? Peut-on déceler des contamina-

le nom secret de la déesse, qui se trouve aussi dans les papyrus magiques. Voir Waegemann 1992; Michel 2004 : 212.

56. Michel $2004: 169, \$ 11.4$. Voir également infra la fig. 10 portant l'inscription grecque : Chnoubis ei, «tu es Chnoubis »). Il faut toutefois relever qu'anoch, du copte ANOK, est parfois une vox magica. Sur son inscription sur les gemmes, voir p. ex. Michel 2001a : $\mathrm{n}^{\text {os }} 505,589$ (= CBd-863, 948). Sur les papyrus : PGM vol. III : 215 , s.v. Anok, Anoch.

57. Michel 2004 : 258-259, $\$$ 11.3.b ; Quack, sous presse : ch. 2.3.3. Pour Bonner 1950 : 168-169, ces gemmes auraient pu servir d'amulettes contre les serpents. Sur le rapport entre Chnoubis et le Dieu d'Israël, voir plus loin. 
tions ou des assimilations de ses caractéristiques chez d'autres divinités et chez d'autres serpents divins? Nous relèverons ici quelques pistes de recherche ${ }^{58}$.

\section{Chnoubis et autres divinités}

La première piste concerne les relations entre Chnoubis et Khnoum. L'homophonie de leurs noms pourrait expliquer une similarité fonctionnelle que révèle leur caractère interchangeable sur les gemmes utérines. Le dieu bélier et le serpent léontocéphale apparaissent dans le même type de scène, dans leur fonction de gardiens de l'utérus-ventouse, mais nous relèverons qu'ils ne sont d'ordinaire pas figurés ensemble, comme s'ils se substituaient l'un à l'autre59.

La deuxième se rapporte à l'appartenance de Chnoubis au réseau des forces salutaires que gouverne Héraclès. Une sardoine inédite de l'Ermitage présente un type unique : une tête juvénile, probablement d'Héraclès, gravée sur sardoine, est entourée de l'inscription Chnoubis ei, "Tu es Chnoubis ». Le pouvoir des mots transforme l'image d'Héraclès en une représentation de Chnoubis. La gemme peut être stylistiquement datée de la période hellénistique, alors que l'inscription, bien qu'antique, semble être postérieure à la gravure ${ }^{60}$.

Une troisième piste soulève la question de la réception et nous mène du côté d'une autre

58. En dehors des amulettes traitées dans ce paragraphe, quelques gemmes contiennent des éléments iconographiques solaires (p. ex. le char du soleil, les bustes d'Hélios et de Séléné), éléments qui renforcent ainsi la signification primaire de Chnoubis à tête radiée. Une gemme du British Museum est, par contre, unique : Hécate est flanquée de Horus et de Chnoubis. Voir Michel 2001a : nº 67 (= CBd-129).

59. Dasen 2002, 2007. Michel 2004 : 334-341 répertorie environ soixante-dix gemmes avec Chnoubis (p. ex. pl. 70, 2-4) et une dizaine avec Khnoum (p. ex. pl. 71, 3). Font exception deux hématites réunissant Chnoubis et Khnoum (337, 54.2.k, pl. 71, $2 ; 338,54.3)$.

60. Ж.6479, inédite. Nous remercions G. Platz pour ses observations sur la gravure de l'inscription. La même inscription, Chnoubis ei, accompagne l'image du serpent léontocéphale sur une gemme de la Collection Skoluda : Michel 2001b : 68-69, n 67. puissance suprême, le Dieu d'Israël. La tradition rabbinique rapporte l'interdiction formelle faite aux juifs de se mettre en contact avec une sorte de deraqôn: "Si l'on trouve une bague avec l'image ... d'un dragon, qu'on la porte à la mer Morte... ". "Quelle est l'espèce de dragon interdite ? Rabbi Siméon ben Éléazar dit : "celui qui a des filaments (ou des écailles) sortant du cou"61. " Les différentes variantes de cette interdiction rabbinique ont été soigneusement analysées par M. Schlüter (Schlüter 1982 : 100-113). Elle a démontré de manière convaincante que cette prescription de la Mishna a été formulée dans la Palestine du $\mathrm{II}^{\mathrm{e}}$ siècle afin de répondre à la question que se posait le juif qui trouvait une bague portant ce motif. Deux cents ans plus tard, cette interdiction est devenue, dans le Talmud babylonien, un motif littéraire et un problème exégétique ${ }^{62}$.

La plupart des chercheurs s'accordent à identifier le mot deraqôn (du grec drakôn) au serpent Chnoubis dont les rayons sont décrits comme des filaments ou des écailles. Cette interprétation a été récemment mise en question par C. Béhar : "L'objection peut être faite que les rayons sortent de la tête de l'animal et non du cou. La représentation d'Agathodaimon nous semble correspondre plutôt à la description rabbinique car Agathodaimon est barbu, et sa barbe clairement indiquée. ${ }^{63}$. À notre avis, le problème herméneutique n'est pas résolu par cette nouvelle proposition, car la barbe ne sort pas non plus du cou.

61. Hadas-Lebel 1979 : 413-420 ; Bohak 2008 : 388-389; Quack, sous presse : ch. 2.3.3.

62. Schlüter 1982 : 108-111. Pour la datation de l'interdiction : «Der mit dieser Tradition verbundene Name wird (wenn man den Namen überhaupt einen Aussagewert beimessen will) - wohl auf Grund der gleichen Vornamen - in der TOSEFTA (und in einer Handschriftenversion des JERUŠALMI) und im JERUŠALMI-Druck unterschiedlich angegeben. Zwischen R. Šimon b. Elazar und R. Šimon b. Azzaj (JERUŠALMI-Druck) liegen zwei Generationen : die Überlieferung dieser Tradition ist also entweder im ersten Drittel oder gegen Ende des zweiten Jahrhunderts anzusetzen ".

63. Béhar $2000: 568$. 


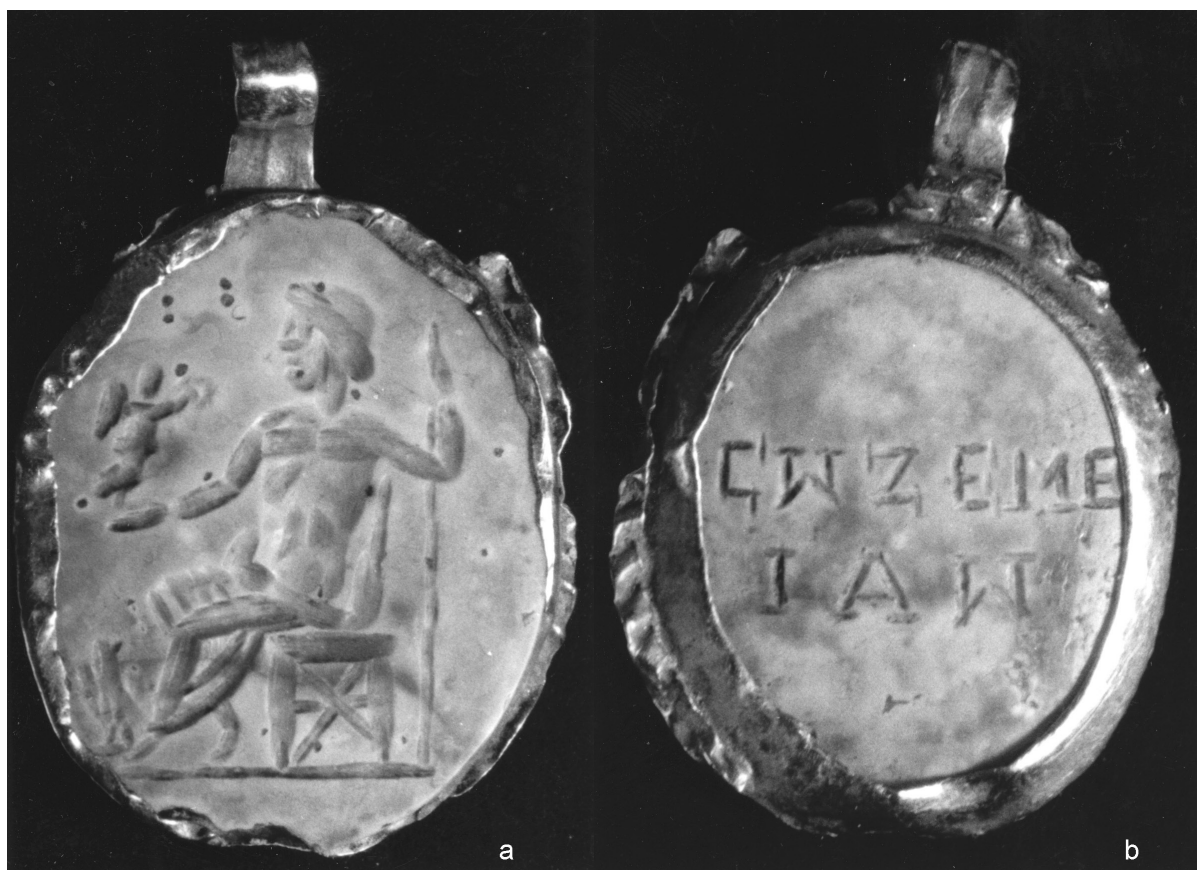

FIG. 7.- Coll. privée. Inscr. : sôze me, Iaô. Photo Claudia Wagner.

Du point de vue iconographique, on pourrait proposer un compromis entre les deux interprétations. En rapprochant le sens du mot tzitzin, "filaments, écailles, touffe " ${ }^{64}$, des types connus de la glyptique impériale, on peut appliquer la description à la crinière du serpent à tête de lion, tout en attribuant une grande importance au fait que les rayons sortent parfois directement du cou du serpent, ce qui n'élimine pas l'interprétation de l'Agathodaimon. Ainsi, l'interdiction concernerait un anneau dont la gravure ne représente pas un serpent ordinaire ${ }^{65}$, mais un serpent à tête de lion ou d'homme - un dieu barbare donc, et tenu pour cette raison par les rabbins pour dangeureux.

Peut-on préciser pourquoi la popularité de Chnoubis constituait un problème pour les rabbins de l'époque impériale ? L'iconographie de

64. Jastrow 1903 : 1279-1280. Nous remercions György Tatár pour la traduction du mot tzitzin.

65. Voir p. ex. Bonner 1950 : D 94 (faisant référence à la gemme quasiment identique publiée dans $S G G 2008$ : Ro 18). quelques gemmes témoigne à leur manière des liens qu'un juif pouvait faire entre Chnoubis et le Dieu d'Israël. L'avers d'une gemme en quartz conservée à Cologne porte l'image de Chnoubis, tandis que le revers est inscrit du nom IAW ${ }^{66}$. Le fabricant de la gemme auraitil réinterprété l'image de Chnoubis en le liant au Dieu d'Israël ? Une gemme conservée dans une collection privée démontre qu'un tel procédé d'interprétation a pu exister ${ }^{67}$. Sur l'avers, Zeus est assis selon le schéma classique qui remonte à Phidias; sur le revers, on lit : sôze me, Iâ̂ (Fig. 7). L'inscription transforme l'image de Zeus sur l'avers en une représentation du Dieu d'Israël, vu d'un œil païen. Une deuxième gemme offre un témoignage du même procédé : une hématite avec Chnoubis sur le revers porte

66. Zwierlein-Diehl 1992: $\mathrm{n}^{\circ}$ 15. Autres gemmes de Chnoubis avec le Trigrammaton : Zazoff 1970 : 237-238, n 162 (Iaô, voces) ; Michel 2001a : 408 (= CBd-779 ; ibis, Iaô) ; Delattel Derchain 1964 : n 64 (signe Chnoubis, Iâo, Remré).

67. Wagner-Boardman 2003 : 42, nº 263. 
sur l'avers l'image de l'Anguipède, un schéma qui représente un des noms du Dieu d'Israël et ne trangresse donc pas la Loi ${ }^{68}$. D'autres gemmes présentent sur l'avers une figure divine, sur le revers le Trigrammaton, témoins probables d'une interprétation païenne ${ }^{69}$.

\section{Chnoubis et LeS AUTRES SERPENTS DIVINS}

Les instigateurs du culte du serpent Glykôn d'Abonuteichos (Ionopolis) ${ }^{70}$, fondé en 150 apr. J.-C., ont-ils récupéré la popularité du Chnoubis des amulettes et assimilé les deux divinités ? Une agate du Cabinet des Médailles semble indiquer que les adeptes de Glykôn pouvaient se représenter leur dieu sous la forme de Chnoubis : la gemme porte gravés les noms Chnoubis, Glykôna et Iầ à côté de l'image de Chnoubis radié et nimbé (Fig. 8) ${ }^{71}$. Cependant, ces rapports furent sans doute fortuits et ne sont à ce jour pas corroborés par d'autres documents. Les descriptions littéraires du serpent Glykôn ne mentionnent ni la tête de lion ni la couronne radiée ou nimbée, des attributs qui restent spécifiques à Chnoubis.

68. Michel 2001a : n⿳0 395 (= CBd-766; Quack, sous presse). Cette pièce appartient à une série de gemmes ornées d'une divinité léontocéphale anguipède et d'une inscription liée au domaine digestif (Michel 2001a : $\mathrm{n}^{\text {os }} 396-400$, = CBd-767-771). Pour l'interprétation ici proposée de l'Anguipède, voir Nagy 2002b. Opinions différentes : Bohak 2008 : 196-198 ; Koenig 2009. Signalons que l'image de Chnoubis est accompagnée une fois de l'acclamation Heis theos Serapis attestant ainsi une autre variante d'interprétation "hénothéiste ": Michel 2001a : n 332 (= CBd-151). Veymiers $2009: 206$ donne la liste d'autres gemmes - représentant Hermès, Zeus ou un foudre posé sur un trône - et où cette acclamation n'accompagne pas l'image de Sérapis. Sur la formule heis theos, voir récemment Belayche 2010.

69. Des gemmes avec l'Anguipède et Iaô, voir p. ex. Michel 2001a : nos 183-186, 201, 209 (= CBd-581-584, 599 et 607).

70. Voir le récit virulent qu'en fait Lucien, Alexandre ou le faux prophète. Sur cette œuvre, dernièrement : Elm von der Osten 2006. Sur Glykôn, voir principalement Robert 1981 (analyse générale) ; Bordenache Battaglia 1988 (catalogue iconographique) ; Alexandrescu-Vianu 2009 : 30-31 (sur la stips retrouvée à Tomis avec la statue en marbre de Glykôn).

71. Delatte/Derchain 1964 : n 82. Cf. Quack, sous presse : ch. 2.3.3 : «Möglicherweise [...] lokale donauländische Arbeit ». Le rapport entre le nominatif Chnoubis et l'accusatif Glykôna reste à interpréter.

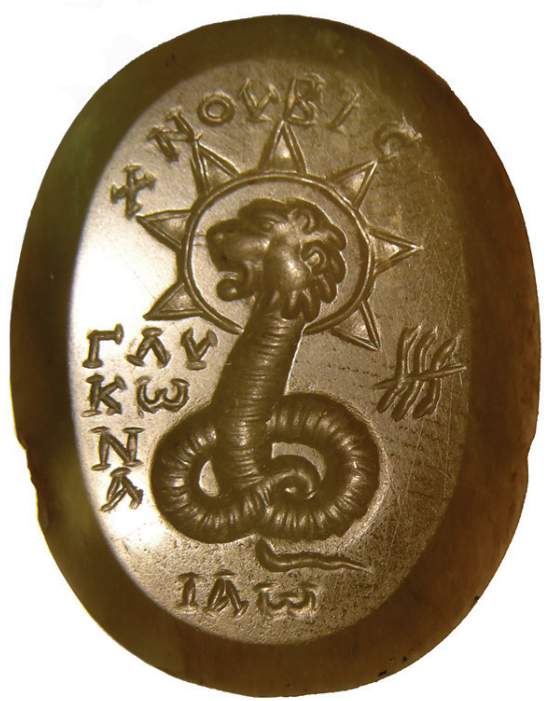

FIg. 8.- Agate brun-vert. Inscr. : Chnoubi, Glykôna, Iaô. Paris, Cabinet des Médailles. Photo A. Mastrocinque.

À l'inverse, plusieurs gemmes signalent l'existence d'un lien possible entre Chnoubis et l'Agathodaimon, ce serpent divin à tête humaine très populaire en Égypte qui personnifie la protection de la fertilité(Dunand 1981). Les intailles avec Chnoubis à tête humaine peuvent faire référence à l'Agathodaimon ${ }^{72}$.

Une troisième piste concerne Asclépios. La popularité de Chnoubis est aussi liée aux nombreuses associations du serpent sacré avec la médecine. Quelques gemmes associent le serpent Chnoubis au dieu Asclépios. Une agate brune du British Museum (Fig. 9a, b) représente sur l'avers Asclépios et Hygie, sur le revers le serpent Chnoubis, associé de manière inhabituelle au nom Chnoum, et non Chnoubis ou Chnoumis, ainsi qu'à son signe qui pourrait désigner la fonction curative de la gemme, l'estomac ${ }^{73}$.

72. Michel $2004: 262-263, \S 11.9$. L'ourobore léontocéphale à tête radiée pourrait-il se rapporter à Chnoubis ?

73. Michel 2001a : no 319 (= CBd-705). Voir encore Michel 2001a : $\mathrm{n}^{\circ} 337$ (= CBd-101), où la forme Chnoum accompagne également l'image du serpent léontocéphale. Une gemme de structure apparentée : Bordenache-Battaglia 1988 : 281, nº 19 (le serpent Glykôn en face d'Asclépios. 
Dasen V. \& Nagy Á. M.

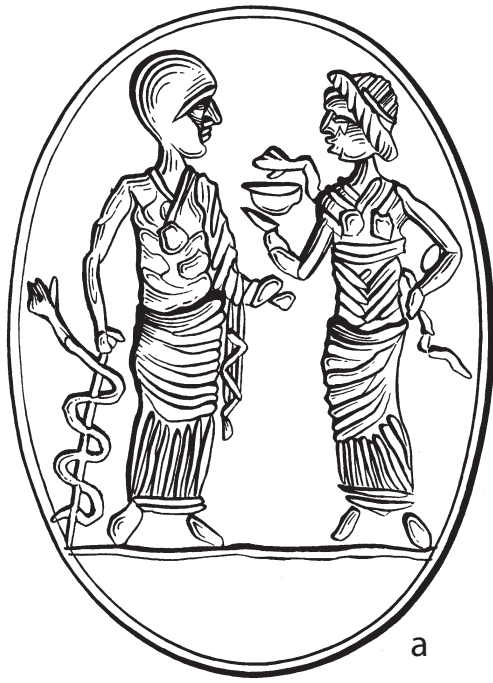

0

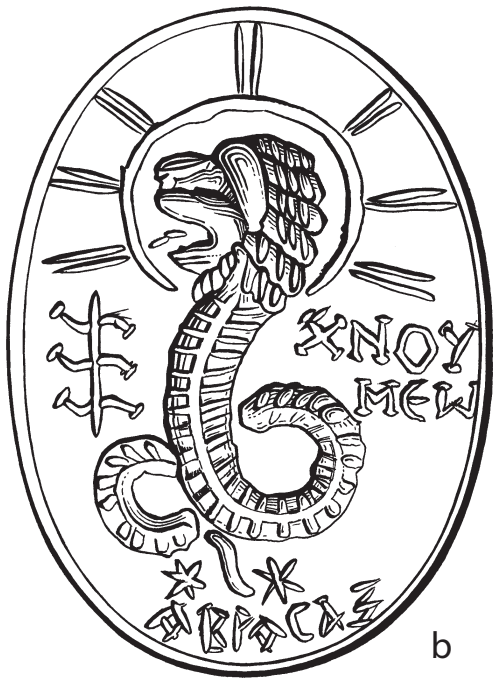

$1 \mathrm{~cm}$

Fig. 9a \& b.- Agate brune. Londres, British Museum inv. G 21, EA 56021.

D'après Michel 2001a : n 319.

Les vertus thérapeutiques attribuées à Chnoubis et à son signe ont-elles bénéficié d'autres associations visuelles ? Quelques gemmes semblent jouer visuellement avec l'image du bâton d'Asclépios autour duquel s'enroule le serpent sacré ; la haste avec les trois serpents que tient un génie léontocéphale sur un jaspe vert du Cabinet des Médailles évoque l'entortillement de l'animal ${ }^{74}$.

Le corpus des amulettes de Chnoubis d'environ 400 pièces apparaît donc remarquablement cohérent : la plupart des gemmes présentent la même combinaison de nom, d'image et de signe sans ajout d'éléments d'autres types. S'ajoute la forme biconvexe qui caractérise la majorité des gemmes de Chnoubis. Cette forme apparaît pour la première fois dans la glyptique hellénistique et devient bientôt très populaire. Plus tard, elle se verra reléguée à l'arrière-plan, sans pour autant être oubliée définitivement, tout en restant rare parmi les

74. Voir p. ex. Delatte/Derchain, $n^{\circ} 303$; Dasen 2011a : 43, fig. 6. gemmes magiques antiques ${ }^{75}$. On peut donc supposer que le matériau et la forme des gemmes de Chnoubis suggéraient ancienneté et prestige ${ }^{76}$.

Le personnage de Chnoubis semble ainsi provenir de l'iconographie des décans égyptiens qui lui conferrent ses propriétés thérapeutiques spécialisées sur la région du ventre et du cœur. Mais $\mathrm{Ch}-$ noubis représente plus qu'un décan. Les inventeurs du Chnoubis des gemmes semblent avoir cherché à créer une divinité solaire qui s'inscrit dans la théologie multiculturelle de l'époque impériale. Sa figure n'est liée à aucune tradition religieuse spécifique. Ses éléments iconographiques, le serpent, la tête de lion radiée et son nom à double signification sont assez généraux pour se prêter à des interprétations différentes -

75. Cette forme caractérise aussi les gemmes gravées d'inscriptions : la surface bombée offre une place optimale pour y graver le texte.

76. Platz 2010. Elle signale que ce type de calcédoine verte translucide (contenant du chrome), coûteuse, était très apprécié dans le milieu de l'élite romaine. 
anciennes et modernes ${ }^{77}$. Son schéma est à la fois conservateur et novateur, un des traits caractéristiques de la magie de l'époque impériale.

\section{ÉLÉMENTS DE CHRONOLOGIE}

Quels résultats pouvons-nous retirer de cette recherche sur le développement de la figure de Chnoubis et sa chronologie?

\section{LES ORIGINES : NÉCHEPSÔ ET LES GEMMES DE Chnoubis}

La plupart des sources offrant une description de l'image de Chnoubis sont postérieures à la production des gemmes, ou au mieux contemporaines. Certaines d'entre elles contiennent des détails, comme les sept rayons ou la protomè de lion, qui durent passer dans la tradition textuelle à partir des gemmes. La seule exception serait le fragment de traité, daté entre 150 et 120 av. J.-C., transmis sous le nom du pharaon Nechepsô (VII ${ }^{e}$ siècle av. J.-C.) cité par Galien (Appendice 5) ${ }^{78}$. Pourtant, la production des gemmes semble avoir commencé au moins cent ans plus tard.

Deux hypothèses pourraient permettre de résoudre cette contradiction. Le témoignage cité par Galien, inconnu des autres sources relatives à l'œuvre de Nechepsô, serait contemporain de l'iconographie des gemmes de Chnoubis ; son auteur aurait emprunté l'identité de Nechepsô pour donner plus d'autorité à sa propre œuvre, un procédé largement attesté ${ }^{79}$. Le texte pourrait cependant dater de l'époque hellénis-

77. Jackson 1985 : 74-108 a bien montré que le schéma Chnoubis se prêtait à la représentation du dieu-créateur du cosmos, Yaldabaôth, décrit dans la variante plus longue de l'Apocryphe de Jean, comme une figure à tête de lion et corps de serpent. Sur la divinité Apedemak, à tête de lion et corps de serpent, voir Török 2011. On remerciera ici László Török d'avoir attiré notre attention sur cette divinité.

78. Hübner $2000: 781$.

79. Turcan 1968 : 397 : «Quand les astrologues gréco-romains invoquent une autorité étrangère, c'est celle d'Hermès, de Néchepso ou de Pétosiris. " tique $^{80}$, mais sans application pratique. Les traités astrologiques étaient des œuvres littéraires théoriques, et ne livraient pas de recettes pour fabriquer des amulettes. Les magoi qui auraient inventé les praxeis de Chnoubis seraient partis de ces savoirs. Il est impossible pour le moment de trancher entre les deux hypothèses.

\section{Chnoubis dans l'ANTIQUité TARdive et À L'ÉPOQUE BYZANTINE}

La popularité de Chnoubis ne s'éteint pas à la fin de l'Antiquité. Plusieurs objets témoignent de sa survie. Trois bracelets byzantins des VI $^{e}$-VII siècles ${ }^{81}$ présentent l'image gravée de Chnoubis. Ils se composent d'un ruban très mince d'argent, avec la gravure des premiers mots du Psaume $90 / 91^{82}$, et sont décorés d'un nombre variable de médaillons représentant des scènes de la vie du Christ. Sur un des médaillons figure le serpent léontocéphale radié. Ces bracelets servaient probablement d'amulettes curatives, comme l'indique le mot Hygieia gravé sur une de ces pièces. Sur une gemme inédite du Cabinet des Médailles est gravée une prière au Seigneur accompagnée d'un signe Chnoubis ${ }^{83}$. Le serpent gravé sur un pendentif en stéatite de l'époque tardive été interprété comme Chnoubis ${ }^{84}$.

Une lamelle-amulette en cuivre, conservée au Musée du Louvre, témoigne du transfert du motif au bas Moyen Âge ${ }^{85}$. Sur le revers (Fig. 10a),

80. Comme l'a dit Bonner $1950: 54:$ «What is important for us is the testimony that this design was recommended in Ptolemaic times for ailments of the stomach. "

81. Maspero 1908 ; Vikan 1991/1992 : fig. 9 ; Kraus 2005 : 55 , $\mathrm{n}^{\text {os }} 55-57$.

82. Sur les versets du Psaume 90/91 utilisés comme amulettes : Kraus 2005.

83. "Seyrig not in Bonner 2 ». La gemme sera publiée par M. Attilio Mastrocinque dans son catalogue sous presse de la collection du Cabinet des Médailles.

84. Vukasović 96. Sur les autres faces du pendentif se trouvent la vox Iaô et les images d'Horus et d'Anubis.

85. Département des antiquités égyptiennes, AF 11704. Voir Ägpten 1996 : 212, n 216 ; Bosson/Aufrère 1999: 203, nº 37. Nous remercions M. Marc Étienne, conservateur du Louvre, d'avoir rendu possible l'étude de la pièce. 


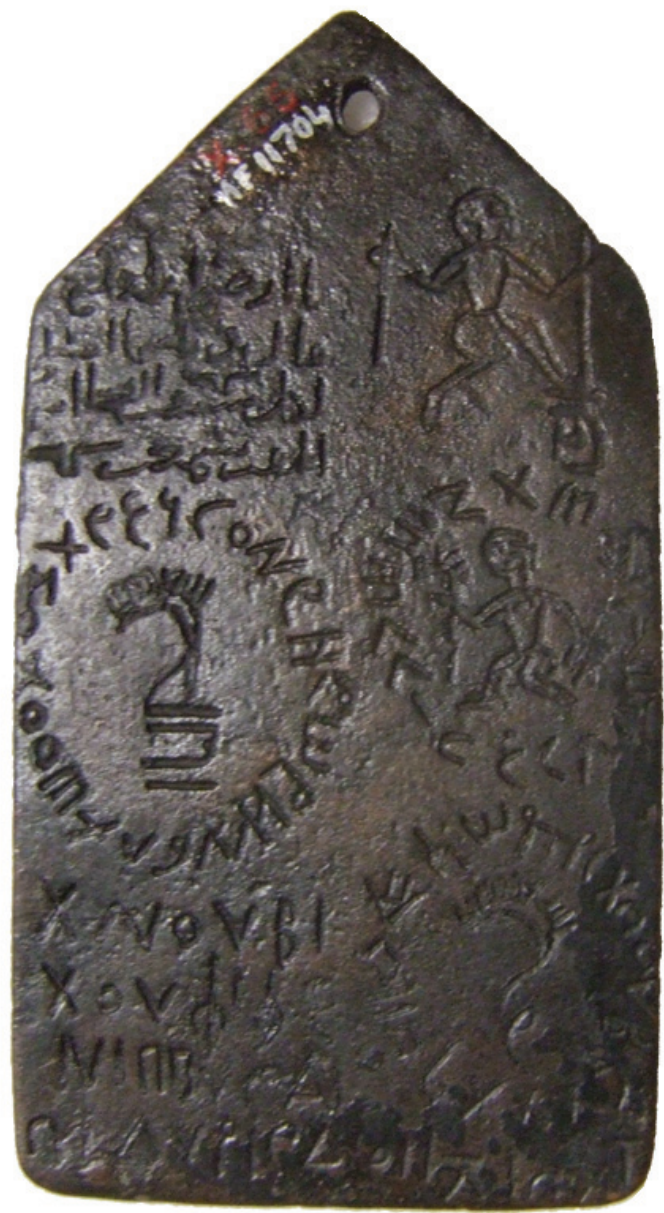

a

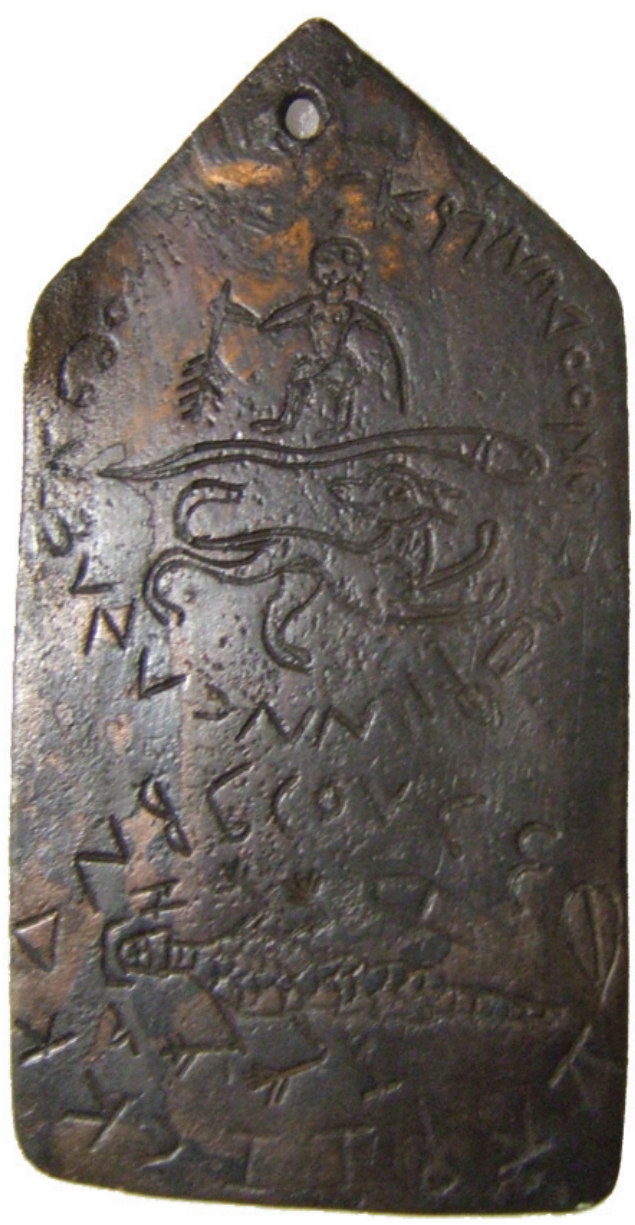

b

FIg. 10.- Lamelle en cuivre. Paris, Musée du Louvre, Département des Antiquités Égyptiennes, inv. AF 11704. Photo D. Bénazeth.

deux cercles composés de lettres grecques et de charactères entourent une figure humaine et différents animaux. Sur l'avers (Fig. 10b) se trouvent un texte arabe, une figure humaine, puis un cercle, enfin des lettres grecques et deux autres cercles. Il est facile d'y reconnaître Chnoubis sur un socle sous la forme d'un serpent lové, la tête couronnée de rayons. On devine le mot Chnoubi dans les lettres grecques gravées à son côté. Le cercle inférieur de l'avers (Fig. 10a) fait référence aux gemmes magiques ophtalmologiques $^{86}$ : comme sur ces pierres, il associe un lézard, la lune et quatre lettres ou signes. Pour dater cette lamelle, le style de la figure de lion a été rapproché de bols magiques arabes du XIII ${ }^{e}$ ou XIV siècle. La lamella de Paris offre ainsi peut-être le premier exemple du transfert d'un motif iconographique propre aux gemmes magiques dans un milieu culturel dont la culture arabe fait partie.

86. Voir la typologie de Michel $2004: \$ 14$. Sur leur utilisation et les références aux sources médicales, Gaillard-Seux 1998. 


\section{CONCLUSION}

Pour l'utilisateur de l'époque romaine impériale, les gemmes de Chnoubis présentaient des traits caractéristiques : une pierre de forme lisse, arrondie, élégante, d'un archaïsme insolite qui faisait référence à un passé lointain, tout comme la figure du serpent combinée à l'allusion aux décans. Ces éléments mettaient en œuvre dans l'imaginaire un savoir ancestral dont l'ancienneté était synonyme d'efficacité. Galien, en dépit de son scepticisme, trouve l'attribution au pharaon-magicien Nechepsô digne d'être mentionnée. Les inventeurs des praxeis surent le faire émerger du monde des décans et le transformer en divinité solaire grâce à l'ajout de sa couronne radiée avec un succès qui témoigne de leur habileté. L'image de cette nouvelle divinité est remarquablement unitaire. Son importance s'explique d'une part par sa fonction guérisseuse et, de l'autre, par sa nature solaire qui l'associe aussi au Dieu d'Israël. Le signe Chnoubis est plus qu'un character quelconque - sur les gemmes magiques il devient quasiment inséparable de Chnoubis.

Parfaitement inséré dans le réseau iconographique des divinités des gemmes magiques, Chnoubis assure une fonction thérapeutique, principalement sur la zone du ventre (estomac, utérus, cœur) et plus largement protectrice.

\section{Appendice}

\section{Hiera Biblos Hermou}

Premier décan du Lion : "(Chnoumos) a une face léonine, émettant des rayons solaires. Le corps entier est celui d'un serpent enroulé en spirale, tourné vers le haut. Il régit les affections produites au cour, kardia. Grave-le sur la pierre agate. " (trad. Ruelle 1908 : 260)

\section{Liber Hermetis Trismegistri}

"Premier décan du Lion (...) Le maître de l'estomac. Un serpent de grande taille couronné de rayons solaires. "
3. Héphestion de Thèbes, Apotelesmatica 4.1 (troisième décan du Cancer) (fin du IV s. ap. J.-C.) "Chnoumis, une protection pour l'estomac. "

\section{Le Lapidaire de Socrate et Denys}

35. "Gravez-y les circonvolutions (speirama) d'un serpent avec l'avant-train ou la tête d'un lion et des rayons. Portée, cette pierre empêche absolument la douleur d'estomac, et quoi que vous mangiez, vous digérerez bien. "

36. "Autre pierre d'onyx. Noire d'aspect dans sa totalité. Elle est utile aux femmes enceintes et à celles qui allaitent. On y grave Chnoubis à trois têtes. » (trad. Halleux \& Schamp 1985)

5. Galien, Sur l'effet des médicaments simples, IX, 2, 19 (Kühn 12, 207, 2-12) (II s. ap. J.-C.)

"Certains attestent pour quelques pierres une propriété comparable à celle qu’a réellement le jaspe vert, qui est utile pour l'œsophage et la bouche de l'estomac, quand il est attaché en amulette. Quelques-uns sertissent aussi la gemme dans une bague et gravent sur elle le serpent ayant des rayons, comme l'a écrit le roi Néchepso dans son quatorzième livre. De cette pierre, moi aussi j'ai une expérience suffisante, et ayant confectionné un petit collier de petites pierres de cette nature, je l'ai fait pendre en amulette du cou en lui donnant une taille telle que les pierres touchent la bouche de l'estomac. Elles n'apparaissent pas moins utiles si elles n'avaient pas l'entaille que Néchepso a décrit. »(trad. Jouanna 2011 : 74-75)

6. Marcellus Empiricus, Sur les remèdes, XX, 98 "Voici un remède contre les maux d'estomac: Grave sur un jaspe un serpent à tête radiée avec sept rayons. Insère la pierre dans une monture d'or et porte-la autour du cou. »

7. Aétius d'Amida, Traité de médecine, II, 18. 1.2.26 (VIe s. ap. J.-C.)

"Le jaspe vert est utile pour l'œsophage et la bouche de l'estomac, attaché en amulette. Certains le sertissent aussi dans des bagues, comme le roi Néchepso l'a écrit. De cette pierre donc moi aussi j'ai une expérience suffisante et ayant confectionné un petit collier fait de pierres de 
cette nature, je l'ai fait pendre en amulette du cou en lui donnant une taille telle que les pierres touchent la bouche de l'estomac. Elles ne paraissaient en rien moins utiles si elles n'avaient pas l'entaille que Néchepso a décrite. " (trad. Jouanna 2011: 63, note 11).

8. Gemmes gravées de la figure de Chnoubis et d'une inscription

8a. Serpentine ou jaspe foncé. Paris, Cabinet des médailles

Delatte/Derchain 1964, no 80.

\section{ФY $\Lambda$ A $\Xi O N$ YГIH $\Sigma$ TOMAXOY ПРОKАOY}

"Garde en bonne santé l'estomac de Proclus. »

8b. Pierre verte (prase, jadéite?). Autrefois coll. privée, Istanboul. Department of Near Eastern Languages, Berkeley, University of California (bientôt déplacée au Hearst Museum, Berkeley).

Bonner 1954, p. 149, n 36, pl. 36.

АПО ТТЕЧАГАІ (pour АПО ТТЕЧАТЕ)

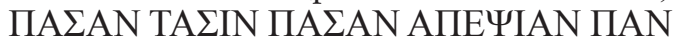
ПONON $\Sigma$ TOMAXOY IOYAIANOY ON ETEKEN NONNA

"Chnoumis, écarte de Julianos, fils de Nonna, toute tension, toute indigestion, toute douleur de l'estomac. »

8c. Jaspe jaune, Londres, British Museum Michel 2001, no 213.

\section{$[\mathrm{XNOY}] \mathrm{BI} \Sigma \Pi \mathrm{E} \Sigma \Sigma \mathrm{E} \Pi \mathrm{\Pi} \Sigma[\Sigma \mathrm{E}$}

"Chnoubis, digère, digère! "

8d. Calcédoine gris-bleu, Michigan. Ann Arbor, University of Michigan, Kelsey Museum of Archaeology.

Bonner 1950, p. 267, D 83.

\section{$\Pi \mathrm{\Pi} \Sigma \Sigma \mathrm{E} \Pi \mathrm{\Pi E} \Sigma \Sigma \mathrm{E}$}

" Digère, digère! »

8e. Calcédoine jaune, Paris, Cabinet des médailles

Delatte/Derchain 1964, no 89.
$\mathrm{H} \Sigma \Omega \Sigma$ IB $\Omega$ EI $\Sigma$ TOMAXOY

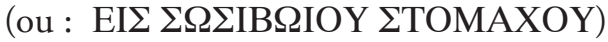

"Pour l'estomac de Sosibios."

8f Hématite, Hambourg, Coll. Skoluda MO85 ; Michel 2001b : pl. 24, no 145.

Michel 2001 b, no 145 .

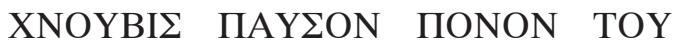
$\Sigma$ TOMAXOY ABPA $\Sigma$ A $\Xi$

"Chnoubis, apaise les maux d'estomac, Abrasax. »

8g Agate-onyx Saint-Pétersbourg, Ermitage, Ж. 1518

Mentionnée par Neverov 2002 : 199.

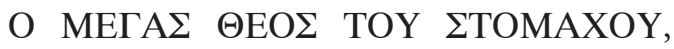
voces

"Le grand dieu de l'estomac »

9. Tosefta V, 1 (traduction M. Hadas-Lebel)

"Si l'on trouve une bague avec l'image de la lune, du soleil ou d'un dragon, qu'on la porte à la mer Morte ; de même pour la figure de la femme qui allaite et de Sérapis. »

"Quelle est l'espèce de dragon interdite? R. Siméon ben Éléazar dit : "celui qui a des filaments (ou des écailles) sortant du cou”."

\section{Abréviations}

ANRW: W. Haase et al. (eds), Aufstieg und Niedergang der römischen Welt (W. de Gruyter, Berlin-New York, 1972 - ).

CBd : The Campbell Bonner database

< http://classics.mfab.hu/talismans/>

LIMC : Lexicon iconographicum mythologiae classicae. Artemis Verlag, Zurich ; Munich ; Düsseldorf 19811999.

GMPT: H. D. Betz (ed.), The Greek Magical Papyri in Translation including the Demotic Spells (The Univ. of Chicago Press, Chicago 1986).

Roscher : Roscher W. H. (ed.), Ausfübrliches Lexikon der griechischen und römischen Mythologie. B.G. Teubner, Leipzig-Berlin, 1884-1937). 
SGG 2004 : A. Mastrocinque (éd.), Sylloge Gemmarum Gnosticarum, I (Istituto poligrafico e Zecca dello Stato/Libreria dello Stato, Rome 2004).

SGG 2008 : A. Mastrocinque (ed.), Sylloge Gemmarum Gnosticarum, II (Istituto poligrafico e Zecca dello Stato/Libreria dello Stato, Rome 2008).

\section{BIBLIOGRAPHIE}

Aвry J.-H. (éd.) 1993.—Les tablettes astrologiques de Grand (Vosges) et l'astrologie en Gaule romaine, actes de la Table-Ronde du 18 mars 1992 au Centre d'études romaines et gallo-romaines de l'Université Lyon III. De Boccard, Paris ; Univ. Lyon III, Lyon.

AA. VV. 1996.- Ägypten, Schätze aus dem Wüstensand. Kunst und Kultur der Christen am Nil. Katalog zur Ausstellung. Gustav-LübckeMuseum Hamm, Museum für Spätantike und Byzantinische Kunst, Staatliche Museen zu Berlin-Preussischer Kulturbesitz, L. Reichert, Wiesbaden.

Alexandrescu-Vianu M. 2009.- The Treasury of Sculptures from Tomis. The Cult Inventory of a Temple. Dacia 53 : 27-46.

BAY C. et al. 2008.- Magie, astrologie et sorcellerie dans l'Antiquité, Musée d'Argentomagus, 4 juillet-21 décembre 2008. Musée d'Argentomagus, Saint-Marcel.

BÉHAR C. 2002.- Les témoignages du culte de Sérapis dans la Palestine romaine et le traité Abodah Zarah. Revue des Études Juives 161 : 567-571.

Belayche N. 2010.- Deus deum ... summorum maximus (Apuleius): Ritual Expressions of Distinction in the Divine World in the Imperial Period, in Mitchell S. \& VAn Nuffelen P. (eds), One God. Pagan Monotheism in the Roman Empire. Cambridge University Press, Cambridge : 141-166.

Вонак G. 2008.—Ancient Jewish Magic. A History. Cambridge University Press, Cambridge.
Bonner C. 1950.- Studies in Magical Amulets Chiefly Graeco-Egyptian. The University of Michigan Press, Ann Arbor.

Bonner C. 1951.- Amulets Chiefly in the British Museum. A Supplementary Article. Hesperia $20: 301-345$.

Bonner C. 1954.- A Miscellany of Engraved Stones. Hesperia 23 : 138-157.

Bordenache-BatTaglia G. 1988.— s.v. Glykon, in LIMC IV : 279-283.

Bosson N. \& Aufrère S. H. 1999.- Égyptes, l'égyptien et le copte, Musée archéologique de Lattes, 3 juin - 31 octobre 1999. Musée archéologique Henri-Prades, Lattes.

Daniel R. W. \& Maltomini F. i990.- Supplementum Magicum I. Papyrologica Coloniensia XVI, 1. Westdeutscher Verlag, Opladen.

DASEN V. 2002.— Métamorphoses de l'utérus, d'Hippocrate à Ambroise Paré. Gesnerus $59: 167-186$.

Dasen V. 2007.- Représenter l'invisible: la vie utérine sur les gemmes magiques, in Dasen V. (éd.), L'embryon humain à travers l'histoire. Images, savoirs et rites. Infolio, Gollion : 41-64.

Dasen V. 2008. - Le secret d'Omphale. Revue Archéologique : 265-281.

DASEN V. 2010.- Des nourrices grecques à Rome?, in Pache Huber V. \& Dasen V. (eds), Politics of Child Care in Historical Perspective. From the World of Wet Nurses to the Networks of Family Child Care Providers. Paedagogica Historica, 46 (6) : 699-713.

Dasen V. (dir.) 2011a.- La médecine à l'époque romaine, Quoi de neuf, docteur? Marchandeau, Lyon.

Dasen V. 2011b.- Magic and Medicine: Gems and the Power of Seals, in Entwistle Chr. \& Adams N. (eds), British Museum Byzantine Seminar, Recent Research on Engraved Gemstones in Late Antiquity, AD 200-600, 28-30 mai 2009. The British Museum Press, Londres : 69-74.

Delatte A. \& Derchain Ph. 1964.- Les intailles magiques gréco-égyptiennes. Bibliothèque nationale, Paris. 
Duffy J. M. (ed.) 1992.- Michaelis Pselli philosophica minora, I, Opuscula logica, physica, allegorica, alia. B. G. Teubner, Leipzig.

DrexLer W. 1890-1897.— s.v. Knuphis, in Roscher II, 1 : col. 1250-1264.

DreXler W. 1924-1937.- s.v. Chnoubis, in ROSCHER VI : col. 859-861.

Dunand F. 1981.- s.v. Agathodaimon, in LIMC I, $1: 276-282$.

Dupont-Sommer A. 1945.- - Le syncrétisme religieux des Juifs d'Éléphantine d'après un ostracon araméen inédit. Revue de l'Histoire des Religions 130 : 17-28.

Dupont-Sommer A. 1947.- 'Yahô' et 'Yahô-seba'ôt' sur des ostraca araméens inédites d'Éléphantine. Comptes rendus de l'Académie des Inscriptions et Belles-Lettres : 75-191.

Elm von der Osten D. 2006.- Die Inszenierung des Betruges und seiner Entlarvung : Divination und ihre Kritiker in Lukians Schrift $<$ Alexandros oder der Lügenprophet>, in ELM VON Der Osten D., RÜPKe J. \& Waldner K. (eds), Texte als Medium und Reflektion von Religion im römischen Reich. Franz Steiner Verlag, Stuttgart :141-157.

Gaillard-Seux P. 1998. - Les maladies des yeux et le lézard vert, in Debru A. \& Sавbан G. (éds), Nommer la maladie. Recherches sur le lexique gréco-latin de la pathologie. PUSE, SaintÉtienne : 93-105.

Gori A. F. 1733-1766.-Museum Florentinum, I-II. ex Typographia Michaelis Nestenus et Francisci Moücke, Florence.

Gourevitch D. 1976. - Les noms latins de l'estomac. Revue de Philologie 50 : 85-110.

Guisan M. 1975.- Bijoux romains d'Avenches. Bulletin de l'association Pro Aventico 23 : 5-39.

GuNDEL W. 1936a.-Dekane und Dekansternbilder. Studien der Bibliothek Warburg 19. Wissenschaftliche Buchgesellschaft, Glückstadt ; Hambourg.

Gundel W. ed. 1936b. - Neue astrologische Texte des Hermes Trismegistos. Funde und Forschungen auf dem Gebiet der antiken Astronomie und Astrologie, Bayerische Akademie der Wissenschaften, Munich.

Gramatopol M. 1974.- Les pierres gravées du Cabinet Numismatique de l'Académie Roumaine. Collection Latomus 138. Latomus, Bruxelles.

Hadas-Lebel M. 1979._- Le paganisme à travers les sources rabbiniques des $\mathrm{II}^{e}$ et $\mathrm{III}^{\mathrm{e}}$ siècles. Contribution à l'étude du syncrétisme dans l'empire romain, in ANRW II 19.2 : 397-485.

Halleux R. \& Schamp J. (éds.) 1985.- Les lapidaires grecs. Les Belles Lettres, Paris.

HübNer W. 2000.— s.v. Nechepso, in Der Neue Pauly $8: 781$.

Jackson H. M. 1985.- The Lion Becomes Man: The Gnostic Leontomorphic Creator and the Platonic Tradition. Scholars Press, Atlanta.

Jounnna J. 2011.- Médecine rationnelle et magie : le statut des amulettes et des incantations chez Galien. Revue des études grecques 124 : 44-77.

KIss Z. 1986. - s.v. Chnoubis, in LIMC, III : 272273.

Koenig Y. 2009.— Des " trigrammes panthéistes " ramessides aux gemmes magiques de l'Antiquité tardive: le cas d'Abrasax, continuité et rupture. Bulletin de l'Institut Français d'Archéologie Orientale 109 : 311-325.

Kotansky R. 1994.- Greek Magical Amulets. The Inscribed Gold, Silver, Copper, and Bronze Lamellae. Papyrologica Coloniensia XXII, 1. Westdeutscher Verlag, Opladen.

Kotansky R., Naveh J. \& Shaked Sh. 1992.- A Greek-Aramaic Silver Amulet from Egypt in the Ashmolean Museum. Le Muséon 105 : 5-26.

Kraus Th. J. 2005.- Septuaginta-Psalm 90 in apotropäischer Verwendung : Vorüberlegungen für eine kritische Edition und (bisheriges) Datenmaterial. Biblische Notizen 125 : 39-73.

Lieven A. von 2000.- Die dritte Reihe der Dekane oder Tradition und Innovation in der spätägyptischen Religion. Archiv für Religionsgeschichte 2 : 21-36. 
MaAskant-Kleibrink M. 1978.- Catalogue of the Engraved Gems in the Royal Coin Cabinet The Hague. Government publishing office, La Haye ; F. Steiner, Wiesbaden.

Maspero J. 1908.- Bracelets-amulettes d'époque byzantine. Annales du Service des Antiquités de l'Égypte 9 : 246-258.

Mastrocinque A. 2002.- Metamorfosi di Kronos su una gemma di Bologna, in MastrocinQue A. (ed.), Atti dell'incontro di studio "Gemme gnostiche e cultura ellenistica", Verona, 22-23 ottobre 1999. Pàtron, Bologne : 103-118.

Mastrocinque A. 2008.- Un'altra imagine transculturale : Chnoubis, in Estienne S. et al. (éds), Image et religion dans l'Antiquité gréco-romaine, actes du colloque de Rome, 11-13 décembre 2003, Centre Jean Bérard, Naples : 391-397.

Michel S. 2001a.- Die magischen Gemmen im Britischen Museum. The British Museum Press, Londres.

Michel S. 2001b.-Bunte Steine - dunkle Bilder : "Magische Gemmen". Biering \& Brinkmann, Munich.

Michel S. 2004.- Die magischen Gemmen. Zu Bildern und Zauberformeln auf geschnittenen Steinen der Antike und Neuzeit. Akademie Verlag, Berlin.

Morigi M. 2006. - Una lamina bilingue dal Medagliere Capitolini : considerazioni sul testo ebraico. Bulletino della Commissione Archeologica Comunale di Roma 107 :163-170.

Mouterde R. 1930-1931.- Le glaive de Dardanos, objets et inscriptions magiques de Syrie. Mélanges de l'université Saint-Joseph Beyrouth $15: 51-139$.

NAGy Á. M. 1992.— Ein Kaiserzeitlicher Talisman, Archäologischer Anzeiger : 99-108.

NAGY Á. M. 2002a.- Gemmae magicae selectae. Sept notes sur l'interprétation des gemmes magiques, in Mastrocinque A. (ed.), Atti dell'incontro di studio "Gemme gnostiche e cultura ellenistica", Verona, 22-23 ottobre 1999. Pàtron, Bologne :153-179.

Nagy Á. M. 2002b._- Puzzling out the Anquipede : Magical Gems and their Relation to Judaism. Journal of Roman Archaeology 15 : 159-172.
NAGY Á. M. sous presse.- Daktylios pharmakités. Magical Healing Gems and Rings in the Graeco-Roman World, in BurnetT CH. \& Csepregi I. (eds), Ritual Healing in Antiquity and the Middle Ages. Sismel, Florence.

NAGY Á. M. 2011._ Magical Gems and Classical Archaeology, in Entwistle Chr., Adams N. (eds), British Museum Byzantine Seminar, Recent Research on Engraved Gemstones in Late Antiquity, $A D$ 200-600, 28-30 mai 2009. The British Museum Press, Londres : 75-81.

Neverov O. 2002.- Les amulettes magiques de l'Ermitage. Essai d'une classification, in Mastrocinque A. (ed.), Atti dell'incontro di studio "Gemme gnostiche e cultura ellenistica", Verona, 22-23 ottobre 1999. Pàtron, Bologne :195-205.

Olivieri A. (ed.) 1935.- - Aetii Amideni Libri medicinales, I-IV. Corpus medicorum graecorum, 8, 1. Teubner, Leipzig; Berlin.

Philipp H. 1986. - Mira et Magica. Gemmen im Ägyptischen Museum der Staatlichen Museen. Preussischer Kulturbesitz, Berlin-Charlottenburg. Ph. von Zabern, Mayence/Rhin.

Plantzos D. 1999.- Hellenistic Engraved Gems. Clarendon Press, Oxford.

Platz G. 2010.- Kleine Praser and ChromiumBearing Chalcedonies. About a Small Group of Engraved Gems. Pallas $83: 179-202$.

Porten B. 1996.- The Elephantine Papyri in English. Three Millennia of Cross-Cultural Continuity and Change. Society of Biblical Literature, Leyde ; New York ; Cologne.

QuACK J. F. sous presse.-Beiträge zu den ägyptischen Dekanen und ihrer Rezeption in der griechischrömischen Welt. Orientalia Lovaniensia Analecta. Louvain.

Reinach S. 1895.— Les pierres gravées. Firmin-Didot, Paris.

Ridder A. DE 1911.- Les bijoux et les pierres gravées. Collection de Clercq, Catalogue VII, 2. E. Leroux, Paris.

Robert L. 1981.- Le serpent Glycon d'Abônoteichos à Athènes et Artémis d'Éphèse à Rome. Comptes rendus de l'Académie des Inscriptions et Belles-Lettres : 513-535. 
Dasen V. \& Nagy Á. M.

Roura C. 1972.- Aprocimaciones al lenguaje cientifico de la coleccion hipocratica. Emerita $40: 319-327$.

Ruelle C. E. 1908.- Hermès Trismégiste, le livre sacré sur les décans. Revue de Philologie de Littérature et d'Histoire Anciennes 32 : 232290.

Sambin Ch. (avec la collaboration de J.-F. CarlotTI) 1995.- - Une porte de fête-sed de Ptolémée II remployée dans le temple de Montou à Médamoud. Bulletin de l'Institut Français d'Archéologie Orientale 95 : 383-457.

SChlüter M. 1982.-Derāqôn und Götzendienst. Studien, ausgehend von $m A Z$ III 3. Peter Lang, Francfort/Main ; Berne.

Sfameni C. 2010.- Magic in Late Antiquity : the Evidence of Magical Gems, in Gwynn D. M. \& Bangert S. (eds), Religious Diversity in Late Antiquity. Brill, Leyde ; Boston : 435473.

Thissen H.-J. 1996. - KMHФ - Ein verkannter Gott. Zeitschrift für Papyrologie und Epigraphik $112: 153-160$.

Töröк L. 2011.- Hellenizing Art in Ancient Nubia 300 BC-AD 250 and its Egyptian Models. Brill, Leyde ; Boston.

TurCan R. 1968.- Littérature astrologique et astrologie littéraire dans l'Antiquité classique. Latomus 27 : 392-405.

Ulmer R. 2010.- The Egyptian Gods in Midrashic Texts. Harvard Theological Review 103 : 181-204.

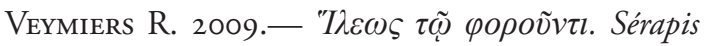
sur les gemmes et les bijoux antiques. Académie royale de Belgique, Bruxelles.
VIKan G. 1991-1992.— Two Byzantine Armbands and the Group to which they belong. Journal of the Walters Art Gallery 49-50 : 33-51.

Vitellozzi P. 2010.-Gemme e cammei della Collezione Guardabassi nel Museo Archeologico Nazionale dell'Umbria a Perugia. Volumnia, Pérouse.

Vismara N. 2000.- Sylloge Nummorum Graecorum. Italia. Milano, Civiche Raccolte Numismatiche VI, Macedonia - Thracia, 3 : Chersonesus Tauricus Sarmatia - Thracia - Chersonesus Thraciae - Isole della Thracia. Civiche raccolte numismatiche, Milan.

Vukasović F. 2010.- Angels and Demons. Jewish Magic through the Ages. Bible Lands Museum, Jérusalem.

Waegeman M. 1992.-A - A $\omega \rho 1 \varphi \rho \alpha \sigma 15$ : Aphrodite's Magical Name. Antiquité Classique 61 : 237-244.

Wagner C. \& Boardman J. 2003.- A Collection of Classical and Eastern Intaglios, Rings, and Cameos. Archaeopress, Oxford.

Zwierlein-Diehl E. 1992.- Magische Amulette und andere Gemmen des Instituts für Altertumskunde der Universität zu Köln. Westdeutscher Verlag, Opladen.

Zwierlein-Diehl E. 2007.—Antike Gemmen und ihr Nachleben. W. de Gruyter, Berlin.

Zazoff P. et al. 1970._-Antike Gemmen in deutschen Sammlungen, III, Braunschweig, Göttingen, Kassel. Prestel Verlag, Munich : 177-272.

Soumis le 12 janvier 2011; accepté le 30 janvier 2011 
Le serpent léontocéphale Chnoubis et la magie de l'époque romaine impériale 